TRANSACTIONS OF THE

AMERICAN MATHEMATICAL SOCIETY

Volume 295, Number 2, June 1986

\title{
CONGRUENCES ON REGULAR SEMIGROUPS
}

\author{
FRANCIS PASTIJN AND MARIO PETRICH
}

\begin{abstract}
Let $S$ be a regular semigroup and let $\rho$ be a congruence relation on $S$. The kernel of $\rho$, in notation $\operatorname{ker} \rho$, is the union of the idempotent $\rho$-classes. The trace of $\rho$, in notation $\operatorname{tr} \rho$, is the restriction of $\rho$ to the set of idempotents of $S$. The pair $(\operatorname{ker} \rho, \operatorname{tr} \rho)$ is said to be the congruence pair associated with $\rho$. Congruence pairs can be characterized abstractly, and it turns out that a congruence is uniquely determined by its associated congruence pair. The triple $((\rho \vee \mathcal{L}) / \mathcal{L}, \operatorname{ker} \rho,(\rho \vee R) / R)$ is said to be the congruence triple associated with $\rho$. Congruence triples can be characterized abstractly and again a congruence relation is uniquely determined by its associated triple.

The consideration of the parameters which appear in the above-mentioned representations of congruence relations gives insight into the structure of the congruence lattice of $S$. For congruence relations $\rho$ and $\theta$, put $\rho T_{l} \theta\left[\rho T_{r} \theta\right.$, $\rho T \theta]$ if and only if $\rho \vee \mathcal{L}=\theta \vee \mathcal{L}[\rho \vee R=\theta \vee R, \operatorname{tr} \rho=\operatorname{tr} \theta]$. Then $T_{l}, T_{r}$ and $T$ are complete congruences on the congruence lattice of $S$ and $T=T_{l} \cap T_{r}$.
\end{abstract}

Introduction and summary. After it was realized by Wagner that a congruence on an inverse semigroup $S$ is uniquely determined by its idempotent classes, Preston provided an abstract characterization of such a family of subsets of $S$ called the kernel normal system (see [2, Chapter 10]). This approach was the only usable means for handling congruences on inverse semigroups for two decades. A new approach to the problem of describing congruences on inverse semigroups was sparked by the work of Scheiblich [13] who described congruences in terms of kernels and traces. A systematic exposition of the achievements of this approach can be found in $[10$, Chapter III].

It was recognized by Feigenbaum [3] that every congruence $\rho$ on a regular semigroup $S$ is uniquely determined by its kernel, $\operatorname{ker} \rho$, equal to elements $\rho$-equivalent to idempotents, and its trace, $\operatorname{tr} \rho$, equal to the restriction of $\rho$ to the set $E(S)$ of idempotents of $S$. In the case of an inverse semigroup $S, \operatorname{ker} \rho$ and $\operatorname{tr} \rho$, as well as their mutual relationship, can be described abstractly by means of simple conditions on a subset of $S$ and an equivalence on $E(S)$ (see [10, Chapter III]). Following in the footsteps of Scheiblich, for orthordox and arbitrary regular semigroups, Feigenbaum [3] and Trotter [14] adopted the following approach: $\operatorname{tr} \rho$ is characterized abstractly and to each such $\operatorname{tr} \rho$ all matching kernels are described. This unbalances the symmetry of the kernel-trace approach by giving preference to the trace. Hence a balanced view relative to the kernel and the trace is evidently called for.

The unqualified success of the kernel-trace approach for inverse semigroups, including its diverse ramifications, gave a certain hope that this may also turn out to be the case for regular semigroups. Judging by the complexity of regular semigroups and the attempts made for both orthodox and general regular semigroups,

Received by the editors November 2, 1984.

1980 Mathematics Subject Classification. Primary 20M10; Secondary 08A30. 
the handling of congruences on regular semigroups must of necessity be more complicated, and thus less explicit, than on inverse semigroups. With this pessimistic forecast, we are actually in for a few pleasant surprises.

After taking care of some preparations in $\S 1$, in $\S 2$ a number of new concepts are introduced. These concepts amount to abstract characterizations of the kernel and the trace of a congruence on a regular semigroup and are used for defining a congruence pair. The climax of this section represents a description of congruences in terms of congruence pairs. Contrary to reasonable expectation that the axioms for a congruence pair ought to be complex, they are actually quite concise but not what one might call explicit.

Certain results in $\S 2$ yield expressions for the least and the greatest congruences with a given trace or kernel. Unlike in inverse semigroups, these do not seem to admit simple expressions in closed form. These deliberations lead in $\S 3$ to the concept of a min network whose basis, it turns out, is of lattice-theoretical nature.

In $\S 4$, the left and the right traces of a congruence are introduced as well as their abstract counterparts, the left and the right normal equivalences. Their mutual relationship and their connection with certain congruences on the congruence lattice are then explored in some detail. Classes of these congruences are intervals of the congruence lattice; their minimum and maximum elements are found explicitly. It is proved that the mapping $\rho \rightarrow \operatorname{tr} \rho$ is a complete homomorphism for several classes of regular semigroups, but the general case remains open.

Various properties of the intervals mentioned above are explored in §5. Modularity of some of these intervals and commutativity of certain congruences are established.

In the final $\S 6$, congruence triples are introduced which are then used to describe an arbitrary congruence. These triples make use of the normal equivalences on the sets $S / \mathcal{L}$ and $S / R$ as well as a normal subset of $S$, which is an abstraction of the kernel. These triples represent a close analogue of admissible triples which are used for describing congruences on a Rees matrix semigroup. For arbitrary regular semigroups they seem the natural setting for an elaborate study of congruences. They admit several natural ramifications thereby providing for unexpected diversity of congruences associated with a given congruence.

1. Preliminaries. In the following we shall use the notation and terminology of [2 and 5]. This will be supplemented with the following.

Throughout the entire paper, $S$ stands for an arbitrary regular semigroup. If $a \in S$, then $V(a)$ denotes the set of inverses of $a$ in $S$. The set of idempotents of $S$ is denoted by $E(S)$. On $E(S)$ we shall consider the relations $\leq_{l}, \leq_{r}$ and $\leq$ given by, for $e, f \in E(S)$,

$$
\begin{aligned}
& e \leq_{l} f \Leftrightarrow e f=e, \\
& e \leq_{r} f \Leftrightarrow f e=e, \\
& e \leq f \Leftrightarrow e f=e=f e .
\end{aligned}
$$

The relation $\leq_{=} \leq_{l} \cap \leq_{r}$ is the natural partial order on $E(S)$. For $e, f \in E(S)$,

$$
S(e, f)=f V(e f) e
$$

is the sandwich set of $e$ and $f$. 
1.1. Result [8, Theorem 1.1, Proposition 2.5]. If $e$ and $f$ are idempotents of $S$, then

$$
\begin{aligned}
S(e, f) & =\{h \in E(S) \mid f h=h=h e \text { and } e f=e h f\} \\
& =\{h \in E(S) \mid f h=h=h e \text { and } h \in V(e f)\} .
\end{aligned}
$$

If $\rho$ is a congruence on $S$ and $h \in S(e, f)$, then $h \rho \in S(e \rho, f \rho)$.

The nice behaviour of idempotents in connection with homomorphisms is further illustrated by the following.

1.2. RESULT [6] (see also [5, LEMMA II.2.7]). If $\rho$ is a congruence on $S$, then $a \rho \in S / \rho$ is an idempotent if and only if a $\rho$ e for some $e \in E(S)$.

The following simple statements will be applied without further mention: for $e, f \in E(S)$,

$$
\begin{aligned}
e \leq_{l} f & \Rightarrow f e \in E(S) \text { and } e \mathcal{L} f e \leq f, \\
e \leq_{r} f & \Rightarrow e f \in E(S) \text { and } e R e f \leq f, \\
e \mathcal{L} f & \Rightarrow S(e, f)=\{f\}, \\
e \mathcal{R} f & \Rightarrow S(e, f)=\{e\} .
\end{aligned}
$$

For a set $X, \mathrm{Eq} X$ is the lattice of equivalence relations on $X$ ordered by inclusion. Further, Con $S$ is the lattice of congruences on $S$. We remark that Con $S$ is a complete sublattice of $\mathrm{Eq} S$. The least element of both $\mathrm{Eq} S$ and $\operatorname{Con} S$ is $\varepsilon$, the equality on $S$, and the greatest element of both $\mathrm{Eq} S$ and $\operatorname{Con} S$ is $\omega$, the universal relation. We shall sometimes use the notation $\varepsilon_{S}, \omega_{S}$ to avoid confusion.

Let $\tau$ be a relation on $S$. The congruence generated by $\tau$ is denoted by $\tau^{*}$ : it is the intersection of all congruences on $S$ which contain $\tau$. If $\gamma$ is an equivalence on $S$, then $\gamma^{0}$ is the greatest congruence on $S$ contained in $\gamma$.

The remaining results in this section are true for arbitrary semigroups.

1.3. RESULT [2, LEMMA 10.3]. If $\gamma$ is an equivalence on $S$, then for $a, b \in S$,

$$
a \gamma^{0} b \Leftrightarrow(x a y) \gamma(x b y) \quad \text { for all } x, y \in S^{1} .
$$

1.4. RESULT. If $₹$ is a family of relations on $S$, then

$$
\bigvee_{\tau \in \mathcal{F}} \tau^{*}=(\bigcup \mathcal{F})^{*}
$$

If $₹$ is a family of equivalences on $S$, then

$$
\bigcap_{\gamma \in \mathcal{F}} \gamma^{0}=\left(\bigcap^{\mathcal{F}}\right)^{0} \text {. }
$$

PROOF. The straightforward argument is omitted.

Let $K \subseteq S$. A congruence $\rho$ on $S$ saturates $K$ if $a \in K$ implies $a \rho \subseteq K$. The greatest congruence on $S$ which saturates $K$ is denoted by $\pi_{K}$.

1.5. RESULT. Let $K \subseteq S$. Then for $a, b \in S, a \pi_{K} b$ if and only if

$$
x a y \in K \Leftrightarrow x b y \in K \quad\left(x, y \in S^{1}\right) .
$$

Define a relation $\theta_{K}$ on $S$ by

$$
a \theta_{K} b \Leftrightarrow a, b \in K \text { or } a, b \in S \backslash K .
$$

Then $\theta_{K} \in \operatorname{Eq} S$ and $\pi_{K}=\theta_{K}^{0}$. 
ProOF. This follows routinely from Result 1.3.

Let $X$ be a set and $\alpha, \beta \in \operatorname{Eq} X$ be such that $\alpha \subseteq \beta$. We define a relation $\beta / \alpha$ on $X / \alpha$ by

Then $\beta / \alpha \in \operatorname{Eq}(X / \alpha)$.

$$
(a \alpha)(\beta / \alpha)(b \alpha) \Leftrightarrow a \beta b \quad(a, b \in X) .
$$

The following results deal with commuting equivalences.

1.6. RESUlt [6, Proposition III.3.8]. If $\lambda, \rho \in \operatorname{Con} S$ are such that $\lambda \subseteq \mathcal{L}$ and $\rho \subseteq R$, then $\lambda \rho=\rho \lambda$. Then

1.7. RESULT. Let $X$ be a set and $\alpha, \beta, \gamma \in \operatorname{Eq} X$ be such that $\alpha \subseteq \beta$ and $\alpha \subseteq \gamma$.

If this is the case, then $\beta \vee \gamma=\beta \gamma$.

$$
(\beta / \alpha)(\gamma / \alpha)=(\gamma / \alpha)(\beta / \alpha) \Leftrightarrow \beta \gamma=\gamma \beta
$$

PROOF. The equivalence of the above statements follows easily from the definitions. The final assertion is proved in [2, Lemma 1.4].

2. Congruence pairs. We begin our study of congruences on a regular semigroup by the consideration of the kernel and the trace of a congruence and their mutual relationship.

2.1. Definition. For a congruence $\rho$ on $S$,

(i) $\operatorname{tr} \rho=\left.\rho\right|_{E(S)}$ is the trace of $\rho$,

(ii) $\operatorname{ker} \rho=\{s \in S \mid s \rho e$ for some $e \in E(S)\}$ is the kernel of $\rho$.

Note that in view of Result 1.2 we have

$$
\operatorname{ker} \rho=\left\{s \in S \mid s \rho s^{2}\right\}
$$

for a congruence $\rho$.

We may thus associate to each congruence $\rho$ on a regular semigroup the ordered pair $(\operatorname{ker} \rho, \operatorname{tr} \rho)$. Our first task is to give an abstract characterization of such a pair and to prove that the pair $(\operatorname{ker} \rho, \operatorname{tr} \rho)$ uniquely determines $\rho$. An abstract characterization of the trace of a congruence was given in [14]. We adopt a different approach here. To this end, we first introduce the following basic concepts.

2.2. Definition. An equivalence $\tau$ on $E(S)$ is normal if $\tau=\operatorname{tr} \tau^{*}$. A subset $K$ of $S$ is normal if $K=\operatorname{ker} \pi_{K}$.

2.3. LEMMA. An equivalence $\tau$ on $E(S)$ is normal if and only if $\tau$ is the trace of a congruence on $S$. If this is the case, then $\tau^{*}$ is the least congruence on $S$ with trace $\tau$.

PROOF. If $\rho$ is a congruence on $S$ with $\tau=\operatorname{tr} \rho$, then $\tau \subseteq \rho$; thus $\tau^{*} \subseteq \rho$, whence $\operatorname{tr} \tau^{*} \subseteq \operatorname{tr} \rho=\tau$. Since $\tau \subseteq \operatorname{tr} \tau^{*}$ trivially holds, it follows that $\operatorname{tr} \tau^{*}=\tau$.

2.4. LEMMA. A subset $K$ of $S$ is normal if and only if $K$ is the kernel of a congruence on $S$. If this is the case, then $\pi_{K}$ is the greatest congruence on $S$ with kernel $K$.

ProOF. If $\rho$ is a congruence on $S$ with $\operatorname{ker} \rho=K$, then $\rho$ saturates $K$. Since $\pi_{K}$ is the greatest congruence saturating $K$ we have $\rho \subseteq \pi_{K}$, whence $K=\operatorname{ker} \rho \subseteq$ $\operatorname{ker} \pi_{K}$. If $k \in \operatorname{ker} \pi_{K}$, then $k \pi_{K} e$ for some $e \in E(S)$. Since $e \in K$ we also have $k \in K$ since $K$ is a union of $\pi_{K}$-classes. Thus $K=\operatorname{ker} \pi_{K}$.

The following simple results show that both the kernel and the trace are compatible with intersections. 
2.5. LEMMA. Let $\mp$ be a nonempty family of congruences on $S$. Then

(i) $\operatorname{ker}\left(\bigcap^{\mathcal{F}}\right)=\bigcap_{\rho \in \mathcal{F}} \operatorname{ker} \rho$,

(ii) $\operatorname{tr}\left(\bigcap^{\mathcal{F}}\right)=\bigcap_{\rho \in \mathcal{F}} \operatorname{tr} \rho$.

ProOF. For any $s \in S$, we have

$$
\begin{aligned}
s \in \operatorname{ker}(\bigcap \mathcal{F}) & \Leftrightarrow s\left(\bigcap^{\mathcal{F}}\right) s^{2} \Leftrightarrow s \rho s^{2} \quad \text { for all } \rho \in \mathcal{F} \\
& \Leftrightarrow s \in \bigcap_{\rho \in \mathcal{F}} \operatorname{ker} \rho,
\end{aligned}
$$

which proves part (i). Furthermore, for any $e, f \in E(S)$, we have

$$
\begin{aligned}
e \operatorname{tr}(\bigcap \mathcal{F}) f & \Leftrightarrow e\left(\bigcap^{\mathcal{F}}\right) f \Leftrightarrow e \rho f \quad \text { for all } \rho \in \mathcal{F} \\
& \Leftrightarrow e\left(\bigcap_{\rho \in \mathcal{F}} \operatorname{tr} \rho\right) f
\end{aligned}
$$

establishing part (ii).

The next lemma will be used many times.

2.6. LEMMA. Let $\rho$ be a congruence on $S$ with $\tau=\operatorname{tr} \rho$.

(i) $(e \rho) R(f \rho)$ in $S / \rho \Leftrightarrow e(\tau R \tau) f$ in $S(e, f \in E(S))$.

(ii) $(a \rho) R(b \rho)$ in $S / \rho \Leftrightarrow a(R \tau R \tau R) b$ in $S(a, b \in S)$.

(iii) $\rho R \rho=R \tau R \tau R=\rho \vee R$,

(iv) $\tau R \tau R \tau=\tau R \tau=\left.(\rho \vee R)\right|_{E(S)}=\tau \vee\left(\left.R\right|_{E(S)}\right)$.

(v) $\tau=\operatorname{tr}(\mathcal{L} \tau \mathcal{L} \tau \mathcal{L} \cap R \tau R \tau R)^{0}=\tau \mathcal{L} \tau \cap \tau R \tau$.

Proof. (i) Let $e, f \in E(S)$ be such that $(e \rho) R(f \rho)$ in $S / \rho$. If $h \in S(e, f)$, then $h \rho \in S(e \rho, f \rho)=\{e \rho\}$ by Result 1.1, so $e \rho h$. Further $h f \in E\left(R_{h}\right)$ and thus $(h f) \rho=(h \rho)(f \rho)=(e \rho)(f \rho)=f \rho$ since $(e \rho) R(f \rho)$. Consequently $(h f) \rho f$. Therefore $e \tau h R(h f) \tau f$ holds in $S$.

If conversely $e \tau g R k \tau f$ in $S$ for some $e, g, k, f \in E(S)$, then in $S / \rho$,

$$
e \rho=(g \rho) R(k \rho)=f \rho .
$$

(ii) If $(a \rho) R(b \rho)$ in $S / \rho$ for $a, b \in S$, then for $a^{\prime} \in V(a), b^{\prime} \in V(b)$, we have

$$
a R\left(a a^{\prime}\right)=e \in E(S), \quad b R\left(b b^{\prime}\right)=f \in E(S)
$$

and

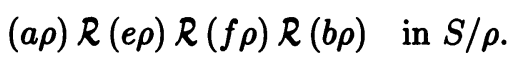

Using part (i) we see that $e(\tau R \tau) f$, whence $a(R \tau R \tau R) b$. $S / \rho$

If conversely $a R e \tau g R h \tau f R b$ in $S$ for $a, b \in S$ and $e, f, g, h \in E(S)$, then in

$$
(a \rho) R(e \rho)=(g \rho) R(h \rho)=(f \rho) R(b \rho),
$$

and thus $(a \rho) R(b \rho)$.

(iii) If $a R_{\tau} R \tau R b$, then by part (ii) we have $(a \rho) R(b \rho)$. Let $e \in E\left(R_{a}\right), f \in$ $E\left(R_{b}\right)$ and $h \in S(e, f)$. From Result 1.1 we have $h \rho \in S(e \rho, f \rho)=\{e \rho\}$ and thus $e \rho h$, whence $e \rho(e h)$. If $a^{\prime} \in V(a)$ with $a a^{\prime}=e$, then $(e h a) a^{\prime}=e h$ since $h e=h$. Therefore $(e h a) R(e h)$, where $(e h) a \rho e a=a$. From Result 1.1 and since $R$ is a left 
congruence, we also have $(e h) R(e h f)=(e f) R(e b)$, where $b=(f b) \rho(e b)$. We have thus proved that $a \rho(e h a) R(e h) R(e b) \rho b$. We conclude that $R \tau R \tau R \subseteq \rho R \rho$.

Conversely, if $a \rho c R d \rho b$, then $a \rho=(c \rho) R(d \rho)=(b \rho)$, and thus by part (ii), $a R \tau R \tau R b$.

It follows from part (ii) that $\rho R \rho$ is an equivalence relation and thus clearly $\rho R \rho=\rho \vee R$ in $\operatorname{Eq} S$.

(iv) By part (i), we have $\tau R \tau \in \operatorname{Eq} E(S)$, so that by transitivity

$$
\tau R \tau=(\tau R \tau)^{2}=\tau R \tau^{2} R \tau=\tau R \tau R \tau
$$

It is easy to see that $\tau R \tau=\tau \vee\left(\left.R\right|_{E(S)}\right)$ in $\operatorname{Eq} E(S)$, whereas $\tau R \tau=\left.(\rho \vee R)\right|_{E(S)}$ is an immediate consequence of parts (i), (ii) and (iii).

(v) Using part (iii) and its dual we have

$$
\rho \subseteq(\rho \vee \mathcal{L}) \cap(\rho \vee \mathcal{R})=\mathcal{L} \tau \mathcal{L} \tau \mathcal{L} \cap R \tau R \tau R
$$

hence

$$
\rho \subseteq(\mathcal{L} \tau \mathcal{L} \tau \mathcal{L} \cap R \tau R \tau R)^{0}
$$

Therefore

$$
\begin{aligned}
\tau & =\operatorname{tr} \rho \subseteq \operatorname{tr}(\mathcal{L} \tau \mathcal{L} \tau \mathcal{L} \cap R \tau R \tau R)^{0} \\
& =\operatorname{tr}\left((\mathcal{L} \tau \mathcal{L} \tau \mathcal{L})^{0} \cap(R \tau R \tau R)^{0}\right) \quad \text { (by Result 1.4) } \\
& =\operatorname{tr}(\mathcal{L} \tau \mathcal{L} \tau \mathcal{L})^{0} \cap \operatorname{tr}(R \tau R \tau R)^{0} \quad \text { (by Lemma 2.5) } \\
& \left.\left.\subseteq(\mathcal{L} \tau \mathcal{L} \tau \mathcal{L})\right|_{E(S)} \cap(R \tau R \tau R)\right|_{E(S)} \\
& =\tau \mathcal{L} \tau \cap \tau R \tau \quad \text { (by (iii) and (iv)). }
\end{aligned}
$$

If $e \tau g R h \tau f$ for some $e, f, g, h \in E(S)$, then $e \rho=(g \rho) R(h \rho)=f \rho$, that is, $(e \rho) R(f \rho)$. From this and its dual we see that $e(\tau \mathcal{L} \tau \cap \tau R \tau) f$ implies that $(e \rho) \not{H}(f \rho)$, whence $e \rho=f \rho$. We have proved that $\tau R \tau \cap \tau \mathcal{L} \tau \subseteq \tau$. Therefore, the desired equalities hold.

We record two more equivalent characterizations of the equivalence $\tau R \tau$.

2.7. LEMMA. Let $\tau$ be the trace of a congruence on $S$. Then for $e, f \in E(S)$, the following are equivalent:

(i) $e \tau R \tau f$,

(ii) $\square \neq(e \tau)(f \tau) \cap E(S) \subseteq f \tau, \square \neq(f \tau)(e \tau) \cap E(S) \subseteq e \tau$,

(iii) $S(e, f) \subseteq e \tau, S(f, e) \subseteq f \tau$.

PROOF. This is immediate from Lemma 2.6 and [14].

2.8. LEMMA. An equivalence $\tau$ on $E(S)$ is normal if and only if $\tau \mathcal{L} \tau \mathcal{L} \tau=$ $\tau \mathcal{L} \tau, \tau R_{\tau} R_{\tau}=\tau R_{\tau}$ and $\tau=\operatorname{tr}\left(\mathcal{L} \tau \mathcal{L} \tau \mathcal{L} \cap R_{\tau} R \tau R\right)^{0}$. If this is the case, then $(\mathcal{L} \tau \mathcal{L} \tau \mathcal{L} \cap R \tau R \tau R)^{0}$ is the greatest congruence on $S$ with trace $\tau$.

PROOF. If $\tau$ is a normal equivalence, then $\tau=\operatorname{tr} \rho$ for some congruence $\rho$ on $S$ by Lemma 2.3. By Lemma 2.6(iv), (v), $\tau$ satisfies the required conditions.

If $\tau \in \operatorname{Eq} E(S)$ with $\tau \mathcal{L} \tau \mathcal{L} \tau=\tau \mathcal{L} \tau$, then

$$
(\mathcal{L} \tau \mathcal{L} \tau \mathcal{L})^{2}=\mathcal{L} \tau \mathcal{L} \tau \mathcal{L}^{2} \tau \mathcal{L} \tau \mathcal{L}=\mathcal{L} \tau \mathcal{L} \tau \mathcal{L} \tau \mathcal{L} \tau \mathcal{L}=\mathcal{L} \tau \mathcal{L} \tau \mathcal{L}
$$

so that $\mathcal{L} \tau \mathcal{L} \tau \mathcal{L}$ is transitive. It is easy to see that $\mathcal{L} \tau \mathcal{L} \tau \mathcal{L}$ is in fact an equivalence. If also $\tau R \tau R \tau=\tau R \tau$ is satisfied, then by duality, $R \tau R \tau R$ is an equivalence. Therefore $\mathcal{L} \tau \mathcal{L} \tau \mathcal{L} \cap R \tau R \tau R$ is an equivalence so that it indeed makes sense to consider the 
congruence $(\mathcal{L} \tau \mathcal{L} \tau \mathcal{L} \cap R \tau R \tau R)^{0}$. If $\tau$ is the trace of this congruence, then $\tau$ is normal by Lemma 2.3 .

If $\rho$ is any congruence with $\tau=\operatorname{tr} \rho$, then by Lemma 2.6(iii),

and thus

$$
\rho \subseteq(\rho \vee \mathcal{L}) \cap(\rho \vee R)=\mathcal{L} \tau \mathcal{L} \tau \mathcal{L} \cap R \tau R \tau R
$$

$$
\rho \subseteq(\mathcal{L} \tau \mathcal{L} \tau \mathcal{L} \cap R \tau R \tau R)^{0} .
$$

2.9. LEMMA. A subset $K$ of $S$ is normal if and only if

$$
K=\operatorname{ker}\left\{\left(x, x^{2}\right) \mid x \in K\right\}^{*} \text {. }
$$

If this is the case, then $\left\{\left(x, x^{2}\right) \mid x \in K\right\}^{*}$ is the least congruence on $S$ with kernel $K$.

PROOF. If $K$ is a normal subset of $S$, then $K=\operatorname{ker} \rho$ for some congruence $\rho$ on $S$ by Lemma 2.4. Clearly $K \subseteq \operatorname{ker}\left\{\left(x, x^{2}\right) \mid x \in K\right\}^{*}$. Further, $\left\{\left(x, x^{2}\right) \mid x \in K\right\} \subseteq \rho$, and thus $\left\{\left(x, x^{2}\right) \mid x \in K\right\}^{*} \subseteq \rho$, whence

$$
\operatorname{ker}\left\{\left(x, x^{2}\right) \mid x \in K\right\}^{*} \subseteq \operatorname{ker} \rho=K,
$$

and the equality prevails.

If conversely $K=\operatorname{ker}\left\{\left(x, x^{2}\right) \mid x \in K\right\}^{*}$, then $K$ is normal by Lemma 2.4 .

2.10. LEMMA. For any congruence $\rho$ on $S, K=\operatorname{ker} \rho, \tau=\operatorname{tr} \rho$ and $a, b \in S$ we have

$$
a \rho b \Leftrightarrow a\left(\mathcal{L} \tau \mathcal{L} \tau \mathcal{L} \cap R_{\tau} \mathcal{R} \tau R\right) b, \quad a b^{\prime} \in K \text { for some }[\text { all }] b^{\prime} \in V(b) .
$$

ProOF. Necessity. If $a \rho b$, then Lemma 2.6(ii) and its dual give $a(R \tau R \tau R) b$ and $a(\mathcal{L} \tau \mathcal{L} \tau \mathcal{L}) b$. Further, if $b^{\prime} \in V(b)$, then $a b^{\prime} \rho b b^{\prime}$, so $a b^{\prime} \in K$.

Sufficiency. Let $a(R \tau R \tau R) b, a,(\mathcal{L} \tau \mathcal{L} \tau \mathcal{L}) b$ and $a b^{\prime} \in K$ for some $b^{\prime} \in V(b)$. Then by Lemma 2.6(ii) and its dual, we have $(a \rho) \not{H}(b \rho)$. Hence $b^{\prime} b \in L_{b} \cap R_{b^{\prime}}$ implies that $\left(b^{\prime} b\right) \rho \in L_{a \rho} \cap R_{b^{\prime} \rho}$ so that by [2, Theorem 2.17],

$$
\left(a b^{\prime}\right) \rho=(a \rho)\left(b^{\prime} \rho\right) \in L_{b^{\prime} \rho} \cap R_{a \rho}=L_{b^{\prime} \rho} \cap R_{b \rho} .
$$

Now $a b^{\prime} \in K$ and the above show that $\left(a b^{\prime}\right) \rho$ is an idempotent in the $\mathcal{H}$-class $L_{b^{\prime} \rho} \cap R_{b \rho}$. Obviously also $\left(b b^{\prime}\right) \rho$ is an idempotent in that $\not{H}$-class, and thus $a b^{\prime} \rho b b^{\prime}$. Therefore,

$$
\begin{array}{cl}
a \rho a b^{\prime} b & \left(\text { since }(a \rho) \mathcal{L}\left(b^{\prime} b\right) \rho\right) \\
\rho b b^{\prime} b & \left(\text { since }\left(a b^{\prime}\right) \rho\left(b b^{\prime}\right)\right) \\
=b . &
\end{array}
$$

2.11. CoROllaRY [3]. A congruence on $S$ is uniquely determined by its kernel and its trace.

Our next basic concept is the following.

2.12. Definition. A pair $(K, \tau)$ is a congruence pair for $S$ if

(i) $K$ is a normal subset of $S$,

(ii) $\tau$ is a normal equivalence on $E(S)$,

(iii) $K \subseteq \operatorname{ker}\left(\mathcal{L} \tau \mathcal{L} \tau \mathcal{L} \cap R_{\tau} R_{\tau} R\right)^{0}$

(iv) $\tau \subseteq \operatorname{tr} \pi_{K}$. 
In such a case we define

$$
\rho_{(K, \tau)}=\pi_{K} \cap(\mathcal{L} \tau \mathcal{L} \tau \mathcal{L} \cap R \tau R \tau R)^{0} .
$$

Note that

$$
\rho_{(K, \tau)}=\left(\mathcal{L} \tau \mathcal{L} \tau \mathcal{L} \cap \theta_{K} \cap R \tau R \tau R\right)^{0}
$$

in view of Results 1.4 and 1.5 .

We are finally ready for the characterization of congruences on a regular semigroup in terms of congruence pairs.

2.13. THEOREM. If $(K, \tau)$ is a congruence pair for $S$, then $\rho_{(K, \tau)}$ is the unique congruence $\rho$ on $S$ for which $\operatorname{ker} \rho=K$ and $\operatorname{tr} \rho=\tau$. Conversely, if $\rho$ is a congruence on $S$, then $(\operatorname{ker} \rho, \operatorname{tr} \rho)$ is a congruence pair for $S$ and $\rho=\rho_{(\operatorname{ker} \rho, \operatorname{tr} \rho)}$.

PROOF. If $(K, \tau)$ is a congruence pair for $S$, then

$$
\begin{aligned}
\operatorname{ker} \rho_{(K, \tau)} & =\operatorname{ker}\left(\pi_{K} \cap(\mathcal{L} \tau \mathcal{L} \tau \mathcal{L} \cap R \tau R \tau R)^{0}\right) \\
& =\operatorname{ker} \pi_{K} \cap \operatorname{ker}(\mathcal{L} \tau \mathcal{L} \tau \mathcal{L} \cap R \tau R \tau R)^{0} \quad(\text { by Lemma 2.5) } \\
& =K \cap \operatorname{ker}\left(\mathcal{L} \tau \mathcal{L} \tau \mathcal{L} \cap R_{\tau} R \tau R\right)^{0} \quad(\text { since } K \text { is normal) } \\
& =K \quad\left(\operatorname{since} K \subseteq \operatorname{ker}(\mathcal{L} \tau \mathcal{L} \tau \mathcal{L} \cap R \tau R \tau R)^{0}\right),
\end{aligned}
$$

and

$$
\begin{aligned}
\operatorname{tr} \rho_{(K, \tau)} & =\operatorname{tr}\left(\pi_{K} \cap\left(\mathcal{L} \tau \mathcal{L} \tau \mathcal{L} \cap R_{\tau} R_{\tau} R\right)^{0}\right) \\
& =\operatorname{tr} \pi_{K} \cap \operatorname{tr}\left(\mathcal{L} \tau \mathcal{L} \tau \mathcal{L} \cap R_{\tau} R_{\tau} R\right)^{0} \quad(\text { by Lemma 2.5) } \\
& =\operatorname{tr} \pi_{K} \cap \tau \quad(\text { by Lemma 2.8) } \\
& =\tau \quad\left(\text { since } \tau \subseteq \operatorname{tr} \pi_{K}\right)
\end{aligned}
$$

The uniqueness of $\rho_{(K, \tau)}$ follows from Corollary 2.11.

If $\rho$ is a congruence, $K=\operatorname{ker} \rho, \tau=\operatorname{tr} \rho$, then $K$ is a normal subset of $S$ by Lemma 2.4 and $\tau$ is a normal equivalence on $E(S)$ by Lemma 2.3. Further, $\rho \subseteq \pi_{K}$ by Lemma 2.4, so $\tau=\operatorname{tr} \rho \subseteq \operatorname{tr} \pi_{K}$. Also $\rho \subseteq(\mathcal{L} \tau \mathcal{L} \tau \mathcal{L} \cap R \tau R \tau R)^{0}$ by Lemma 2.8, and thus $K=\operatorname{ker} \rho \subseteq \operatorname{ker}(\overline{\mathcal{L}} \tau \mathcal{L} \tau \mathcal{L} \cap R \tau R \tau R)^{0}$. Hence $(K, \tau)$ is a congruence pair and by the above $\operatorname{ker} \rho=\operatorname{ker} \rho_{(K, \tau)}, \operatorname{tr} \rho=\operatorname{tr} \rho_{(K, \tau)}$. From Corollary 2.11 we thus have $\rho=\rho_{(K, \tau)}$.

Note that the condition $\tau \subseteq \operatorname{tr} \pi_{K}$ can be written more explicitly as follows:

$$
e \tau f, \quad x, y \in S^{1}, \quad x e y \in K \Rightarrow x f y \in K .
$$

A simpler expression for $\rho_{(K, \tau)}$ is provided by the following result.

2.14. COROLLARY. If $(K, \tau)$ is a congruence pair for $S$, then for any $a, b \in S$,

$$
a \rho_{(K, \tau)} b \Leftrightarrow a\left(\mathcal{L} \tau \mathcal{L} \tau \mathcal{L} \cap R_{\tau} R \tau R\right) b, \quad a b^{\prime} \in K \text { for some }[\text { all }] b^{\prime} \in V(b) .
$$

PROOF. This follows directly from Lemma 2.10 and Theorem 2.13.

We now record an obvious consequence of the above deliberations.

2.15. PROPOSITION. Let $\operatorname{Cp} S$ be the poset of all congruence pairs for $S$ under the partial order given by

$$
(K, \tau) \leq\left(K^{\prime}, \tau^{\prime}\right) \Leftrightarrow K \subseteq K^{\prime}, \tau \subseteq \tau^{\prime} .
$$


Then the mappings

$$
\rho \rightarrow(\operatorname{ker} \rho, \operatorname{tr} \rho), \quad(K, \tau) \rightarrow \rho_{(K, \tau)}
$$

are mutually inverse isomorphisms of $\operatorname{Con} S$ and $\mathrm{Cp} S$.

3. The min network. In this section we show how our consideration of kernels and traces of congruences on $S$ naturally gives rise to certain sublattices of Con $S$.

3.1. NotATION. If $\rho$ is a congruence on $S, \operatorname{ker} \rho=K, \operatorname{tr} \rho=\tau$, then we put

$$
\begin{aligned}
& \rho_{T}=\tau^{*}, \quad \rho^{T}=\left(\mathcal{L} \tau \mathcal{L} \tau \mathcal{L} \cap R_{\tau} R_{\tau} R\right)^{0}, \\
& \rho_{K}=\left\{\left(x, x^{2}\right) \mid x \in K\right\}^{*}, \quad \rho^{K}=\pi_{K} .
\end{aligned}
$$

We summarize the main points of Lemmas 2.3, 2.4, 2.8 and 2.9 in

3.2. THEOREM. Let $\rho$ be a congruence on $S$. Then $\rho_{T}, \rho^{T}\left[\rho_{K}, \rho^{K}\right]$ are, respectively, the least and the greatest congruences with trace [kernel] equal to $\operatorname{tr} \rho[\operatorname{ker} \rho]$.

The same results concerning $\rho_{T}$ and $\rho^{K}$ were given in $[9, \S 3]$, which also contains another characterization of $\rho^{T}$ and $\rho_{K}$.

3.3. CoROllary [9, ThEOREM 3.2]. If $\rho$ and $\theta$ are congruences on $S$, then

$$
\begin{gathered}
\operatorname{tr} \rho \subseteq \operatorname{tr} \theta \Rightarrow \rho_{T} \subseteq \theta_{T}, \quad \rho^{T} \subseteq \theta^{T}, \\
\operatorname{ker} \rho \subseteq \operatorname{ker} \theta \Rightarrow \rho_{K} \subseteq \theta_{K} .
\end{gathered}
$$

Recall that a transformation $x \rightarrow x^{*}$ on a poset $P$ is a closure operation if for all $x, y \in P$,

(i) $x \leq y$ implies $x^{*} \leq y^{*}$,

(ii) $x^{*} \leq x$,

(iii) $\left(x^{*}\right)^{*}=x^{*}$.

The following result concerns closure operations on lattices.

3.4. LEMMA. Let $L$ be a lattice and $x \rightarrow x^{*}$ and $x \rightarrow x^{+}$be closure operations on $L$ such that

$$
x^{*}=y^{*}, \quad x^{+}=y^{+} \Rightarrow x=y \quad(x, y \in L) .
$$

Then for all $x \in L$,

$$
x^{*} \wedge x^{+}=\left(x^{*}\right)^{+} \vee\left(x^{+}\right)^{*} \text { and } x=x^{*} \vee x^{+}
$$

Proof. Since $x^{*} \leq x$, we have $\left(x^{*}\right)^{+} \leq x^{+}$. Also $\left(x^{+}\right)^{*} \leq x^{+}$and thus $\left(x^{*}\right)^{+} \vee\left(x^{+}\right)^{*} \leq x^{+}$. Similarly, $\left(x^{*}\right)^{+} \vee\left(x^{+}\right)^{*} \leq x^{*}$ and hence

$$
\left(x^{*}\right)^{+} \vee\left(x^{+}\right)^{*} \leq x^{*} \wedge x^{+} \text {. }
$$

It follows that

$$
\left(x^{*}\right)^{+} \leq\left(x^{*}\right)^{+} \vee\left(x^{+}\right)^{*} \leq x^{*} \wedge x^{+} \leq x^{*}
$$

Since ${ }^{+}$is a closure operation, we obtain

$$
\left(x^{*}\right)^{+}=\left(\left(x^{*}\right)^{+} \vee\left(x^{+}\right)^{*}\right)^{+}=\left(x^{*} \wedge x^{+}\right)^{+} .
$$

Also,

$$
\left(x^{+}\right)^{*} \leq\left(x^{*}\right)^{+} \vee\left(x^{+}\right)^{*} \leq x^{*} \wedge x^{+} \leq x^{+}
$$


and since ${ }^{*}$ is a closure operation,

$$
\left(x^{+}\right)^{*}=\left(\left(x^{*}\right)^{+} \vee\left(x^{+}\right)^{*}\right)^{*}=\left(x^{*} \wedge x^{+}\right)^{*} .
$$

Hence $\left(x^{*}\right)^{+} \vee\left(x^{+}\right)^{*}=x^{*} \wedge x^{+}$.

For $x \in L$, we have $x^{+} \leq x^{+} \vee x^{*} \leq x$ and since we deal with a closure operation, $x^{+}=\left(x^{+} \vee x *\right)^{+}$. Similarly, we get $x^{*}=\left(x^{+} \vee x^{*}\right)^{*}$. Thus $x=x^{+} \vee x^{*}$.

We are now able to give a new proof of [9, Theorems 3.3 and 3.4].

3.5. THEOREM. If $\rho$ is a congruence on $S$, then

$$
\rho_{T} \cap \rho_{K}=\rho_{T K} \vee \rho_{K T}, \quad \rho=\rho_{T} \vee \rho_{K}=\rho^{T} \cap \rho^{K} .
$$

PROOF. From Corollary 3.3 it easily follows that $\rho \rightarrow \rho_{T}$ and $\rho \rightarrow \rho_{K}$ are closure operations on the lattice $\operatorname{Con} S$. If $\rho, \theta \in \operatorname{Con} S$, then

$$
\begin{aligned}
\rho_{T}=\theta_{T}, \rho_{K}=\theta_{K} & \Leftrightarrow \operatorname{tr} \rho=\operatorname{tr} \theta, \operatorname{ker} \rho=\operatorname{ker} \theta \\
& \Leftrightarrow \rho=\theta \quad \text { (by Corollary 2.11). }
\end{aligned}
$$

We can thus apply Lemma 3.4 to obtain the results involving $\rho_{T}$ and $\rho_{K}$. That $\rho=\rho^{T} \cap \rho^{K}$ follows from Theorem 2.13.

In view of the foregoing theorem we may, as in [9], consider the sublattice of Con $S$ which consists of the congruences $\rho, \rho_{T}, \rho_{K}, \rho_{T K}, \rho_{K T}, \ldots$ This lattice is called the min network corresponding to $\rho$.

4. The left and the right traces. We shall introduce here some relations on the lattice Con $S$. They will turn out to be complete congruences induced by certain complete homomorphisms of Con $S$ into $\operatorname{Eq} E(S)$.

4.1. Definition. For a congruence $\rho$ on $S, \operatorname{ltr} \rho=\operatorname{tr}(\rho \vee \mathcal{L})^{0}$ is the left trace of $\rho, \operatorname{rtr} \rho=\operatorname{tr}(\rho \vee R)^{0}$ is the right trace of $\rho$.

An abstract characterization of left and right traces will be given by means of the following concepts.

4.2. Definition. An equivalence $\tau$ on $E(S)$ is

(i) left normal if $\tau \mathcal{L} \tau \mathcal{L} \tau=\tau \mathcal{L} \tau$ and $\tau=\operatorname{tr}(\mathcal{L} \tau \mathcal{L} \tau \mathcal{L})^{0}$,

(ii) right normal if $\tau R \tau R \tau=\tau R \tau$ and $\tau=\operatorname{tr}(R \tau R \tau R)^{0}$.

The next result will be used several times.

4.3. LEMMA. Let $\rho$ be a congruence on $S, \tau=\operatorname{tr} \rho, \tau_{l}=\operatorname{ltr} \rho$ and $\tau_{r}=\operatorname{rtr} \rho$.

(i) $R \tau R \tau R=\rho \vee R=(\rho \vee R)^{0} \vee R=R \tau_{r} R \tau_{r} R$.

(ii) $\tau R \tau=\tau_{r} R \tau_{r}$.

(iii) $\tau_{r}=\operatorname{tr}(R \tau R \tau R)^{0}=\operatorname{tr}\left(R \tau_{r} R \tau_{r} R\right)^{0}=\operatorname{rtr}(R \tau R \tau R)^{0}=\operatorname{rtr}\left(R \tau_{r} R \tau_{r} R\right)^{0}$.

(iv) $\tau=\tau_{l} \cap \tau_{r}$.

PROOF. (i) In view of Lemma 2.6(iii), it suffices to show that $\rho \vee R=(\rho \vee R)^{0} \vee R$. Since obviously $\rho \subseteq(\rho \vee R)^{0}$, we have $\rho \vee R \subseteq(\rho \vee R)^{0} \vee R$. On the other hand $(\rho \vee R)^{0} \subseteq \rho \vee R$ and $R \subseteq \rho \vee R$ give $(\rho \vee R)^{0} \vee R \subseteq \rho \vee R$.

(ii) From Theorem 2.6(iv) and part (i), we find that $\tau R \tau=\left.(\rho \vee R)\right|_{E(S)}=$ $\left.\left((\rho \vee R)^{0} \vee R\right)\right|_{E(S)}=\tau_{r} R \tau_{r}$

(iii) This follows directly from part (i).

(iv) From Result 1.4, Lemma 2.5 and Lemma 2.6(v) we have

$$
\begin{aligned}
\tau & =\operatorname{tr}(\mathcal{L} \tau \mathcal{L} \tau \mathcal{L} \cap R \tau R \tau R)^{0} \\
& =\operatorname{tr}(\mathcal{L} \tau \mathcal{L} \tau \mathcal{L})^{0} \cap \operatorname{tr}(R \tau R \tau R)^{0}=\tau_{l} \cap \tau_{r} .
\end{aligned}
$$


4.4. LEMMA. An equivalence $\tau_{r}$ on $E(S)$ is right normal if and only if it is the right trace of a congruence on $S$. If this is the case, then $\left(R \tau_{r} R \tau_{r} R\right)^{0}$ is the greatest congruence on $S$ with right trace $\tau_{r}$.

PROOF. If $\tau_{r}$ is the right trace of a congruence $\rho$, then $\tau_{r}=\operatorname{tr}(\rho \vee R)^{0}$, so that by Lemma 2.6(iv), we have $\tau_{r} R \tau_{r} R \tau_{r}=\tau_{r} R \tau_{r}$. Using Lemma 4.3(iii), we may conclude that $\tau_{r}$ is a right normal equivalence.

Suppose conversely that $\tau_{r}$ is a right normal equivalence on $E(S)$. Then $\tau_{r} R \tau_{r} R \tau_{r}$ $=\tau_{r} R \tau_{r}$ so that $R \tau_{r} R \tau_{r} R$ is an equivalence on $S$ and $\tau_{r}=\operatorname{tr}\left(R \tau_{r} R \tau_{r} R\right)^{0}$. By Lemma 2.6(iii), we obtain

$$
\left(R \tau_{r} R \tau_{r} R\right)^{0} \vee R=R \tau_{r} R \tau_{r} R
$$

so that

$$
\tau_{r}=\operatorname{tr}\left(\left(R \tau_{r} R \tau_{r} R\right)^{0} \vee R\right)^{0}=\operatorname{rtr}\left(R \tau_{r} R \tau_{r} R\right)^{0} .
$$

If $\rho$ is any congruence with $\operatorname{rtr} \rho=\tau_{r}$, then $\rho \subseteq(\rho \vee R)^{0}=\left(R \tau_{r} R \tau_{r} R\right)^{0}$, $\operatorname{tr}\left(R \tau_{r} R \tau_{r} R\right)^{0}=\tau_{r}$ by Lemma 4.3. Thus $\left(R \tau_{r} R \tau_{r} R\right)^{\overline{0}}$ is the greatest congruence with right trace $\tau_{r}$.

4.5. COROLlARY. An equivalence $\tau$ on $E(S)$ is normal if and only if it is the intersection of a left normal equivalence and a right normal equivalence.

PROOF. If $\tau$ is a normal equivalence, then by Lemma 2.3 there exists a congruence $\rho$ on $S$ with $\operatorname{tr} \rho=\tau$. By Lemma 4.4 and its dual, $\pi=\operatorname{ltr} \rho$ is a left normal equivalence and $\tau_{r}=\operatorname{rtr} \rho$ is a right normal equivalence. By Lemma 4.3(iv), we have $\tau=\tau_{l} \cap \tau_{r}$

If $\tau=\tau_{l} \cap \tau_{r}$ where $\tau_{l}$ is a left normal and $\tau_{r}$ a right normal equivalence, then $\mathcal{L} \tau_{l} \mathcal{L} \tau_{l} \mathcal{L}$ and $R \tau_{r} R \tau_{r} R$ are equivalences on $S$ and

$$
\begin{aligned}
\tau & =\operatorname{tr}\left(\mathcal{L} \tau_{l} \mathcal{L} \tau_{l} \mathcal{L}\right)^{0} \cap \operatorname{tr}\left(R \tau_{r} R \tau_{r} R\right)^{0} \\
& =\operatorname{tr}\left(\mathcal{L} \tau_{l} \mathcal{L} \tau_{l} \mathcal{L} \cap R \tau_{r} R \tau_{r} R\right)^{0} \quad \text { (by Result } 1.4 \text { and Lemma 2.5), }
\end{aligned}
$$

so that $\tau$ is a normal equivalence by Lemma 2.3 .

We now introduce several relations on Con $S$.

4.6. Notation. For any $\rho, \theta \in \operatorname{Con} S$, let

$$
\begin{aligned}
\rho T_{l} \theta & \Leftrightarrow \operatorname{lt} \rho=\operatorname{lt} \theta, \\
\rho T_{r} \theta & \Leftrightarrow \operatorname{rtr} \rho=\operatorname{rtr} \theta, \\
\rho T \theta & \Leftrightarrow \operatorname{tr} \rho=\operatorname{tr} \theta, \\
\rho K \theta & \Leftrightarrow \operatorname{ker} \rho=\operatorname{ker} \theta .
\end{aligned}
$$

4.7. THEOREM. The mapping

$$
\left.\rho \rightarrow(\rho \vee R)\right|_{E(S)} \quad(\rho \in \operatorname{Con} S)
$$

is a complete lattice homomorphism of Con $S$ into $\mathrm{Eq} E(S)$ which induces $T_{r}$.

ProOF. Let $₹$ be a family of congruences on $S$, and suppose that $e, f \in E(S)$ with $e\left(\left(\bigvee_{\rho \in \mathcal{F}} \rho\right) \vee R\right) f$. Then $e\left(\bigvee_{\rho \in \mathcal{F}}(\rho \vee R)\right) f$ and there exist $x_{0}, \ldots, x_{n} \in S$ and $\rho_{1}, \ldots, \rho_{n} \in \mathcal{F}$ such that

$$
e=x_{0}\left(\rho_{1} \vee R\right) x_{1}\left(\rho_{2} \vee R\right) \cdots x_{n-1}\left(\rho_{n} \vee R\right) x_{n}=f
$$


For every $1 \leq i \leq n$ we know from Lemma 2.6(iii) that

$$
\rho_{i} \vee R=R\left(\operatorname{tr} \rho_{i}\right) R\left(\operatorname{tr} \rho_{i}\right) R,
$$

so there exist idempotents $g_{i-1}, h_{i} \in E(S)$ such that

$$
x_{i-1} R g_{i-1}\left(\operatorname{tr} \rho_{i}\right) R\left(\operatorname{tr} \rho_{i}\right) h_{i} R x_{i}
$$

and we find

$$
\begin{array}{r}
e R g_{0}\left(\operatorname{tr} \rho_{1}\right) R\left(\operatorname{tr} \rho_{1}\right) h_{1} R g_{1}\left(\operatorname{tr} \rho_{2}\right) R\left(\operatorname{tr} \rho_{2}\right) \\
\cdots h_{n-1} R g_{n-1}\left(\operatorname{tr} \rho_{n}\right) R\left(\operatorname{tr} \rho_{n}\right) h_{n} R f .
\end{array}
$$

Thus $e\left(\bigvee_{\rho \in \mathcal{F}}\left(\operatorname{tr} \rho \vee\left(\left.R\right|_{E(S)}\right)\right)\right) f$, which by Lemma 2.6(iv) yields

$$
e\left(\left.\bigvee_{\rho \in \mathcal{F}}(\rho \vee R)\right|_{E(S)}\right) f
$$

We have proved that

$$
\left.\left(\left(\bigvee_{\rho \in \mathcal{F}} \rho\right) \vee R\right)_{\mid E(S)} \subseteq \bigvee_{\rho \in \mathcal{F}}(\rho \vee R)\right|_{E(S)}
$$

and the reverse inclusion obviously holds.

Let $e, f \in E(S)$ be such that $e\left(\bigcap_{\rho \in \mathcal{F}}(\rho \vee R)\right) f$. Then for every $\rho \in \mathcal{F}$, we have $e(\rho \vee R) f$. Thus, if $g \in S(e, f)$ we have from Theorem 2.6(iv) and Theorem 2.7 that $g \rho e$ for all $\rho \in \mathcal{F}$. Further, $g f \in E\left(R_{g}\right)$ and $(g f) \rho=(g \rho)(f \rho)=(e \rho)(f \rho)=f \rho$ for all $\rho \in \mathcal{F}$ since $(e \rho) R(f \rho)$ by Lemma 2.6(ii), (iii). Consequently

that is,

$$
e\left(\bigcap_{\rho \in \mathcal{F}} \rho\right) g R g f\left(\bigcap_{\rho \in \mathcal{F}} \rho\right) f
$$

Hence

$$
e\left(\left(\bigcap_{\rho \in \mathcal{F}} \rho\right) \vee R\right) \mid E(S) f
$$

$$
\left.\left(\left(\bigcap_{\rho \in \mathcal{F}} \rho\right) \vee R\right)_{\mid E(S)} \supseteq \bigcap_{\rho \in \mathcal{F}}(\rho \vee R)\right|_{E(S)}
$$

holds and the reverse inclusion obviously holds.

We have proved that the mapping $\left.\rho \rightarrow(\rho \vee R)\right|_{E(S)}$ is a complete lattice homomorphism of $\operatorname{Con} S$ into $\operatorname{Eq} E(S)$. For $\rho, \theta \in \operatorname{Con} S$, we have

$$
\begin{aligned}
\left.(\rho \vee R)\right|_{E(S)} & =\left.(\theta \vee R)\right|_{E(S)} \\
& \Leftrightarrow(\operatorname{tr} \rho) R(\operatorname{tr} \rho)=(\operatorname{tr} \theta) R(\operatorname{tr} \theta) \quad(\text { by Lemma 2.6(iv) }) \\
& \Rightarrow \operatorname{tr}(R(\operatorname{tr} \rho) R(\operatorname{tr} \rho) R)^{0}=\operatorname{tr}(R(\operatorname{tr} \theta) R(\operatorname{tr} \theta) R)^{0} \\
& \Rightarrow \rho T_{r} \theta \quad(\text { by Lemma 2.6(iii)) } \\
& \Rightarrow(\operatorname{tr} \rho) R(\operatorname{tr} \rho)=(\operatorname{tr} \theta) R(\operatorname{tr} \theta) \quad(\text { by Lemma 4.3(ii)) } \\
& \left.\Leftrightarrow(\rho \vee R)\right|_{E(S)}=\left.(\theta \vee R)\right|_{E(S)} \quad(\text { by Lemma 2.6(iv)). }
\end{aligned}
$$

Hence

$$
\left.(\rho \vee R)\right|_{E(S)}=\left.(\theta \vee R)\right|_{E(S)} \Leftrightarrow \rho T_{r} \theta
$$


4.8. COROLlaRY. We have $T=T_{l} \cap T_{r}$ and

$$
\rho \rightarrow\left(\left.(\rho \vee \mathcal{L})\right|_{E(S)},\left.(\rho \vee R)\right|_{E(S)}\right) \quad(\rho \in \operatorname{Con} S)
$$

is a complete lattice homomorphism of $\operatorname{Con} S$ into $(\mathrm{Eq} E(S))^{2}$ which induces $T$.

Proof. For $\rho, \theta \in \operatorname{Con} S$,

$$
\begin{aligned}
\rho T \theta \Leftrightarrow \operatorname{tr} \rho & =\operatorname{tr} \theta \\
\Rightarrow \operatorname{trt} \rho & =\operatorname{tr}(\mathcal{R}(\operatorname{tr} \rho) \mathcal{R}(\operatorname{tr} \rho) \mathcal{R})^{0} \\
& =\operatorname{tr}(\mathcal{R}(\operatorname{tr} \theta) \mathcal{R}(\operatorname{tr} \theta) \mathcal{R})^{0}=\operatorname{rtr} \theta \\
\operatorname{ltr} \rho & =\operatorname{tr}(\mathcal{L}(\operatorname{tr} \rho) \mathcal{L}(\operatorname{tr} \rho) \mathcal{L})^{0} \\
& =\operatorname{tr}(\mathcal{L}(\operatorname{tr} \theta) \mathcal{L}(\operatorname{tr} \theta) \mathcal{L})^{0}=\operatorname{ltr} \theta \\
\Rightarrow \rho\left(T_{1}\right. & \left.\cap T_{r}\right) \theta \quad(\text { by Lemma 4.3(iii) and its dual) } \\
\Rightarrow & \operatorname{tr} \rho=\operatorname{rtr} \rho \cap \operatorname{ltr} \rho=\operatorname{rtr} \theta \cap \operatorname{lt} \theta=\operatorname{tr} \theta
\end{aligned}
$$

(by Lemma 4.3(iv))

$$
\Rightarrow \rho T \theta \text {. }
$$

Hence $T=T_{l} \cap T_{r}$. The remaining part in the statement of the corollary now follows easily from Theorem 4.7.

4.9. CoROllaRY (CF. $[4,7,12]$ ). The relations $T_{l}, T_{r}$ and $T$ are complete congruences on $\operatorname{Con} S$.

We will need a result concerning complete congruences on complete lattices.

4.10. LEMMA. Let $L$ be a complete lattice and $\rho$ be a complete congruence on L. For $x \in L$,

$$
\bar{x}=\bigwedge_{z \in x \rho} z
$$

is the least element in $x \rho$ and for any $A \subseteq L$,

$$
\bigvee_{x \in A} \bar{x}=\bigvee_{x \in A} x
$$

PROOF. That $\bar{x}$ is the least element in $x \rho$ is clear from the fact that $\rho$ is a complete congruence. Therefore also

$$
\bigvee_{x \in A} \bar{x} \in\left(\bigvee_{x \in A} x\right) \rho
$$

and thus

$$
\bigvee_{x \in A} x \leq \bigvee_{x \in A} \bar{x}
$$

If $y \in A$, then in $L / \rho$

$$
y \rho \leq\left(\bigvee_{x \in A} x\right) \rho=\overline{\left(\bigvee_{x \in A} x\right)} \rho
$$


so that

It follows that

$$
y \rho=\left(y \wedge \overline{\left(\bigvee_{x \in A} x\right)}\right) \rho .
$$

whence

$$
\bar{y} \leq y \wedge \overline{\left(\bigvee_{x \in A} x\right)}
$$

and the equality

$$
\bar{y} \leq \overline{\bigvee_{x \in A} x}
$$

holds.

$$
\bigvee_{x \in A} \bar{x}=\overline{\bigvee_{x \in A} x}
$$

The following supplements the notation introduced in 3.1 .

4.11. NOTATION. If $\rho$ is a congruence on $S$, we put

$$
\begin{array}{cl}
\rho_{T_{l}}=\left(\leq_{r} \cap \rho\right)^{*}, & \rho_{T_{r}}=\left(\leq_{l} \cap \rho\right)^{*}, \\
\rho^{T_{l}}=(\rho \vee \mathcal{L})^{0}, & \rho^{T_{r}}=(\rho \vee R)^{0} .
\end{array}
$$

The next two results describe the classes of the congruences $T_{r}$ and $T$.

4.12. THEOREM. If $\rho \in \operatorname{Con} S$, then $\rho T_{r}$ is the interval $\left[\rho_{T_{r}}, \rho^{T_{r}}\right]$ of Con $S$. For any family $\mathcal{F}$ of congruences on $S$, we have

$$
\left(\bigvee_{\rho \in \mathcal{F}} \rho\right)_{T_{r}}=\bigvee_{\rho \in \mathcal{F}} \rho_{T_{r}}, \quad\left(\bigcap_{\rho \in \mathcal{F}} \rho\right)^{T_{r}}=\bigcap_{\rho \in \mathcal{F}} \rho^{T_{r}} .
$$

ProOF. Clearly $\left(\leq_{l} \cap \rho\right)^{*} \subseteq \rho$, so that

$$
\left.\left.\left(\left(\leq_{l} \cap \rho\right)^{*} \vee R\right)\right|_{E(S)} \subseteq(\rho \vee R)\right|_{E(S)}
$$

Let $e, f \in E(S)$ be such that $e \rho \vee R f$. Let $h \in S(e, f)$. By Lemmas 2.6(iv) and 2.7, we have $h \in e \rho$. Thus $e\left(\leq_{l} \cap \rho\right) h$. Further, $h R h f \leq f$ and

$$
(h f) \rho=(h \rho)(f \rho)=(e \rho)(f \rho)=f \rho
$$

since $(e \rho) R(f \rho)$ by Lemma 2.6(i), (iii). We thus have

$$
e\left(\leq_{l} \cap \rho\right) h R(h f)\left(\leq_{l} \cap \rho\right) f
$$

and hence

$$
e\left(\operatorname{tr}\left(\leq_{l} \cap \rho\right)^{*}\right) R\left(\operatorname{tr}\left(\leq_{l} \cap \rho\right)^{*}\right) f
$$

so by Lemma 2.6(iv), we have

$$
e\left(\left(\leq_{l} \cap \rho\right)^{*} \vee R\right) f
$$

We conclude that the equality

$$
\left.\left(\left(\leq_{l} \cap \rho\right)^{*} \vee R\right)\right|_{E(S)}=\left.(\rho \vee R)\right|_{E(S)}
$$

holds. By Theorem 4.7 we have $\left(\leq_{l} \cap \rho\right)^{*} T_{r} \rho$. Hence $\left(\leq_{l} \cap \rho\right)^{*}$ is indeed the least element in $\rho T_{r}$. The remaining statements of the theorem are now clear from Lemmas 4.3, 4.4, Corollary 4.9 and Lemma 4.10.

With the notation of $\S 3$ we obtain in a similar way the following result. 
4.13. THEOREM. If $\rho$ is a congruence on $S$, then $\rho T$ is the interval $\left[\rho_{T}, \rho^{T}\right]$ of Con $S$. For any family $\mathcal{F}$ of congruences on $S$,

$$
\left(\bigvee_{\rho \in \mathcal{F}} \rho\right)_{T}=\bigvee_{\rho \in \mathcal{F}} \rho_{T}, \quad\left(\bigcap_{\rho \in \mathcal{F}} \rho\right)^{T}=\bigcap_{\rho \in \mathcal{F}} \rho^{T} .
$$

The following result establishes certain relationships among $\rho_{T}$ and $\rho^{T}$ with their one-sided counterparts.

4.14. THEOREM. Let $\rho$ be a congruence on $S$. Then

$$
\rho_{T}=\rho_{T_{l}} \vee \rho_{T_{r}}, \quad \rho^{T}=\rho^{T_{l}} \cap \rho^{T_{r}} .
$$

ProOF. By Corollaries 4.8 and 4.9, $(\operatorname{Con} S) / T$ is a complete lattice and $T_{l} / T$, $T_{r} / T$ are complete lattice congruences on $(\operatorname{Con} S) / T$ such that $T_{l} / T \cap T_{r} / T$ is the equality on $(\operatorname{Con} S) / T$. Clearly, for $\rho \in \operatorname{Con} S, \rho_{T_{l}} T\left[\rho_{T_{r}} T\right]$ is the least element in the $\left(T_{l} / T\right)$-class $\left[\left(T_{r} / T\right)\right.$-class $]$ of $\rho T$.

For $\rho, \theta \in \operatorname{Con} S$, we obtain

$$
\begin{aligned}
\rho T \leq \theta T \quad \text { in }(\operatorname{Con} S) / T & \Leftrightarrow(\rho \cap \theta) T \rho \\
& \Rightarrow \rho_{T} \subseteq \rho \cap \theta \subseteq \theta \\
& \Rightarrow \rho_{T T_{r}} \subseteq \theta_{T_{r}} \quad(\text { by Theorem 4.12) } \\
& \Rightarrow \rho_{T_{r}} \subseteq \theta_{T_{r}} \quad\left(\text { since } \rho_{T} T_{r} \rho\right) \\
& \Rightarrow \rho_{T_{r}} T \leq \theta_{T_{r}} T .
\end{aligned}
$$

Therefore $\rho T \rightarrow \rho_{T_{r}} T$ is a closure operation on $(\operatorname{Con} S) / T$. Similarly for the mapping $\rho T \rightarrow \rho_{T_{l}} T$.

If $\rho, \theta \in \operatorname{Con} S$ are such that $\rho_{T_{l}} T=\theta_{T_{l}} T$ and $\rho_{T_{r}} T=\theta_{T_{r}} T$, then

$$
\rho T_{l} \rho_{T_{l}} T \theta_{T_{l}} T_{l} \theta, \quad \rho T_{r} \rho_{T_{r}} T \theta_{T_{r}} T_{r} \theta
$$

and hence $\rho T=\theta T$ since $T=T_{l} \cap T_{r}$ by Corollary 4.8. We can thus use Lemma 3.4 to obtain

$$
\rho T=\rho_{T_{l}} T \vee \rho_{T_{r}} T
$$

so that

$$
\left(\rho_{T_{l}} \vee \rho_{T_{r}}\right) T \rho
$$

and thus by Theorem 4.13 and $T \subseteq T_{l}, T \subseteq T_{r}$, we get

$$
\rho_{T}=\left(\rho_{T_{l}} \vee \rho_{T_{r}}\right)_{T}=\rho_{T_{l} T} \vee \rho_{T_{r} T}=\rho_{T_{l}} \vee \rho_{T_{r}}
$$

We further have

$$
\begin{aligned}
\rho^{T_{l}} \cap \rho^{T_{r}} & =(\rho \vee \mathcal{L})^{0} \cap(\rho \vee R)^{0} \\
& =((\rho \vee \mathcal{L}) \cap(\rho \vee R))^{0} \quad(\text { by Result 1.4 }) \\
& =\rho^{T} \quad(\text { by Lemma 2.6(iii) }) .
\end{aligned}
$$

The next two results concern commutativity of certain congruences. 
4.15. THEOREM. Let $\rho$ be a congruence on $S$. Then every congruence in $\left[\rho_{T}, \rho^{T_{l}}\right]$ commutes with every congruence in $\left[\rho_{T}, \rho^{T_{r}}\right]$.

PROOF. Let $\alpha \in\left[\rho_{T}, \rho^{T_{l}}\right]$ and $\beta \in\left[\rho_{T}, \rho^{T_{r}}\right]$. We have $\rho_{T} T \rho T_{r} \rho^{T_{r}}$ and thus $\rho_{T} T_{r} \rho^{T_{r}}$ in view of $T \subseteq T_{r}$. Therefore $\rho_{T} T_{r} \beta$ so that, in view of Lemma 4.3(ii),

$$
\left(\operatorname{tr} \rho_{T}\right) R\left(\operatorname{tr} \rho_{T}\right)=(\operatorname{tr} \beta) R(\operatorname{tr} \beta)
$$

and hence

$$
R\left(\operatorname{tr} \rho_{T}\right) R\left(\operatorname{tr} \rho_{T}\right) R=R(\operatorname{tr} \beta) R(\operatorname{tr} \beta) R .
$$

Consequently Lemma 2.6(ii) gives

$$
\begin{aligned}
a \beta b & \Rightarrow a R(\operatorname{tr} \beta) R(\operatorname{tr} \beta) R b \\
& \Leftrightarrow a R\left(\operatorname{tr} \rho_{T}\right) R\left(\operatorname{tr} \rho_{T}\right) R b \\
& \Leftrightarrow\left(a \rho_{T}\right) R\left(b \rho_{T}\right)
\end{aligned}
$$

for any $a, b \in S$. Thus $\beta / \rho_{T}$ is a congruence contained in the $R$-relation on $S / \rho_{T}$. Dually, $\alpha / \rho_{T}$ is contained in the $\mathcal{L}$-relation on $S / \rho_{T}$. By Results 1.6 and 1.7, we have $\alpha \beta=\beta \alpha$.

4.16. THEOREM. Let $\rho$ be a congruence on $S$. Then $\rho^{T_{l}}$ commutes with $\rho^{T_{r}}$ and $\rho_{T_{l}}$ commutes with $\rho_{T_{r}}$.

ProOF. The first statement is a direct consequence of Theorem 4.15. As we have seen in the proof of Theorem 4.14, the mappings

$$
\rho T \rightarrow \rho_{T_{l}} T, \quad \rho T \rightarrow \rho_{T_{r}} T \quad(\rho \in \operatorname{Con} S)
$$

are closure operations on $(\operatorname{Con} S) / T$ which satisfy the conditions of Lemma 3.4. Thus by Lemma 3.4, we get

$$
\rho_{T_{l}} T \wedge \rho_{T_{r}} T=\rho_{T_{r} T_{l}} T \wedge \rho_{T_{l} T_{r}} T
$$

whence

$$
\rho_{T_{r} T_{l}} \subseteq \rho_{T_{l}} \cap \rho_{T_{r}} \subseteq \rho_{T_{r}}, \quad \rho_{T_{l} T_{r}} \subseteq \rho_{T_{l}} \cap \rho_{T_{r}} \subseteq \rho_{T_{l}}
$$

Therefore

$$
\rho_{T_{r}} T_{l}\left(\rho_{T_{l}} \cap \rho_{T_{r}}\right) T_{r} \rho_{T_{l}}
$$

so that

and dually

$$
\rho_{T_{r}} \in\left[\left(\rho_{T_{l}} \cap \rho_{T_{r}}\right)_{T},\left(\rho_{T_{l}} \cap \rho_{T_{r}}\right)^{T_{l}}\right]
$$

$$
\rho_{T_{l}} \in\left[\left(\rho_{T_{l}} \cap \rho_{T_{r}}\right)_{T},\left(\rho_{T_{l}} \cap \rho_{T_{r}}\right)^{T_{r}}\right] .
$$

The desired result now follows from Theorem 4.15.

4.17. COROLlaRY. Let $\rho$ be a congruence on $S$. Then $\rho_{T}=\rho_{T_{l}} \rho_{T_{r}}=\rho_{T_{r}} \rho_{T_{l}}$.

Proof. This is immediate from Theorems 4.14 and 4.16.

4.18. COROLLARY. An equivalence $\tau$ on $E(S)$ is normal if and only if

$$
\left.\left(\leq_{l} \cap \tau\right)^{*}\left(\leq_{r} \cap \tau\right)^{*}\left(\leq_{r} \cap \tau\right)^{*}\left(\leq_{l} \cap \tau\right)\right)^{*}
$$

and

$$
\tau=\operatorname{tr}\left(\leq_{l} \cap \tau\right)^{*}\left(\leq_{r} \cap \tau\right)^{*}
$$


PROOF. If $\tau$ is normal, then $\tau=\operatorname{tr} \rho$ for some $\rho \in \operatorname{Con} S$ by Lemma 2.3. The first part of the statement of the corollary holds by Corollary 4.17. Also the second part follows from Corollary 4.17 since $\tau=\operatorname{tr} \rho_{T}$.

Conversely, if $\tau \in \mathrm{Eq} E(S)$ is such that $\left(\leq_{l} \cap \tau\right)^{*}$ and $\left(\leq_{r} \cap \tau\right)^{*}$ commute, then $\left(\leq_{l} \cap \tau\right)^{*}\left(\leq_{r} \cap \tau\right)^{*} \in$ Con $S$. If, moreover, $\tau$ is the trace of this congruence, then it is normal by Lemma 2.3 .

With a congruence $\rho$ on $S$ we have associated several new ones. The situation is depicted by Diagram 1 .

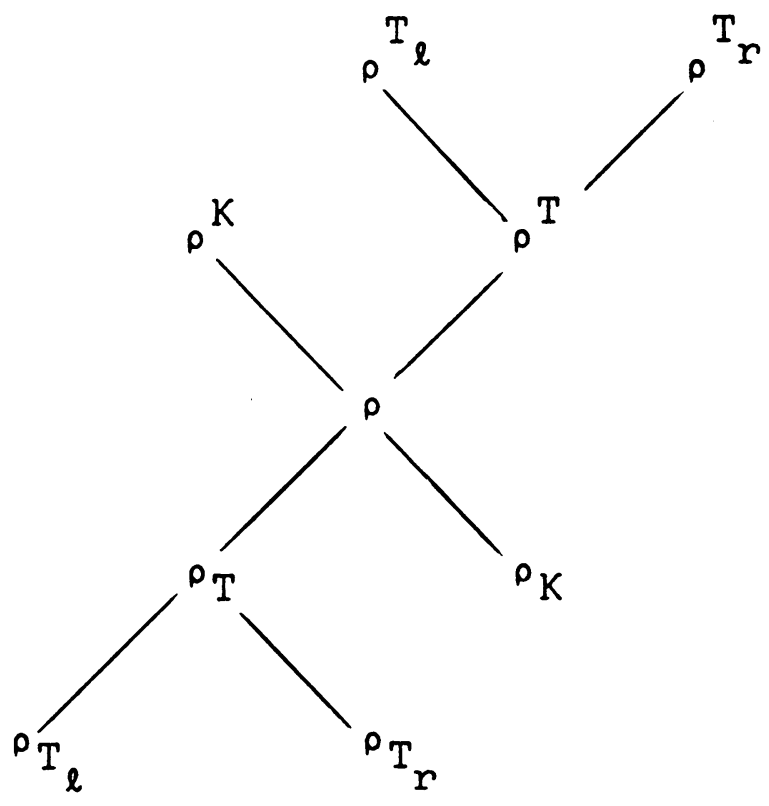

Diagram 1

4.19. Problem. Obviously

$$
\rho \rightarrow \operatorname{tr} \rho \quad(\rho \in \operatorname{Con} S)
$$

is an order-preserving mapping of Con $S$ into $\mathrm{Eq} E(S)$. By Lemma 2.5, this mapping is a complete $n$-homomorphism inducing $T$ on $\operatorname{Con} S$. The question arises whether this mapping is a complete lattice homomorphism, or equivalently, whether the normal equivalences on $E(S)$ form a complete sublattice of $\mathrm{Eq} E(S)$. In order for this to be true it suffices that for every nonempty family $\mathcal{F}$ of congruences

$$
\operatorname{tr}(\bigvee \mathcal{F})=\bigvee_{\rho \in \mathcal{F}} \operatorname{tr} \rho
$$

holds, where the latter join is to be taken in $\operatorname{Eq} E(S)$. As we shall see below this property is satisfied in some large classes of regular semigroups.

Recall that a semigroup $S$ is called group bound if for every $a \in S$ there exists a natural number $n$ such that $a^{n}$ belongs to a subgroup of $S$. We note that group bound regular semigroups include completely regular semigroups and finite regular semigroups. A regular semigroup $S$ is said to be locally inverse if for every $e \in$ $E(S), e S e$ is an inverse semigroup. 
4.20. THEOREM. Let $S$ be either locally inverse, group bound or orthodox. Then

$$
\rho \rightarrow \operatorname{tr} \rho \quad(\rho \in \operatorname{Con} S)
$$

is a complete homomorphism of $\operatorname{Con} S$ into $\operatorname{Eq} E(S)$ which induces $T$.

PROOF. Let $\mathcal{F}$ be a family of congruences on $S$. Clearly $\bigvee_{\rho \in \mathcal{F}} \operatorname{tr} \rho \leq \operatorname{tr} \bigvee_{\rho \in \mathcal{F}} \rho$ always holds. If for $e, f \in E(S)$ we have $e\left(\operatorname{tr} \bigvee_{\rho \in \mathcal{F}} \rho\right) f$, then there exist $\rho_{1}, \ldots, \rho_{n} \in$ F and $x_{0}, \ldots, x_{n} \in S$ such that

$$
e=x_{0} \rho_{1} x_{1} \rho_{2} x_{2} \cdots x_{n-1} \rho_{n} x_{n}=f .
$$

We first assume that $S$ is locally inverse. For $i=0, \ldots, n$, we choose $x_{i}^{\prime} \in V\left(x_{i}\right)$. Since $S$ is locally inverse, sandwich sets are singletons [8, Theorem 7.6]. Thus, there exist elements $h_{0}, \ldots, h_{n}$ such that $S\left(x_{i}^{\prime} x_{i}, x_{i} x_{i}^{\prime}\right)=\left\{h_{i}\right\}$ for $i=0, \ldots, n$. We note that $h_{0}=e$ and $h_{n}=f$. For any $i$ we have $x_{i-1} \rho_{i}=x_{i} \rho_{i}$, so

$$
\left(\left(x_{i-1}^{\prime} x_{i-1}\right) \rho_{i}\right) \mathcal{L}\left(x_{i-1} \rho_{i}\right)=\left(x_{i} \rho_{i}\right) \mathcal{L}\left(\left(x_{i}^{\prime} x_{i}\right) \rho_{i}\right)
$$

and

$$
\left(\left(x_{i-1} x_{i-1}^{\prime}\right) \rho_{i}\right) R\left(x_{i-1} \rho_{i}\right)=\left(x_{i} \rho_{i}\right) R\left(\left(x_{i} x_{i}^{\prime}\right) \rho_{i}\right) .
$$

Using Result 1.1 we have

$$
S\left(\left(x_{i-1}^{\prime} x_{i-1}\right) \rho_{i},\left(x_{i-1} x_{i-1}^{\prime}\right) \rho_{i}\right)=S\left(\left(x_{i}^{\prime} x_{i}\right) \rho_{i},\left(x_{i} x_{i}^{\prime}\right) \rho_{i}\right),
$$

where

$$
h_{i-1} \rho_{i} \in S\left(\left(x_{i-1}^{\prime} x_{i-1}\right) \rho_{i},\left(x_{i-1} x_{i-1}^{\prime}\right) \rho_{i}\right)
$$

and

$$
h_{i} \rho_{i} \in S\left(\left(x_{i}^{\prime} x_{i}\right) \rho_{i},\left(x_{i} x_{i}^{\prime}\right) \rho_{i}\right) .
$$

Since locally inverse semigroups are closed for taking homomorphic images, $S / \rho_{i}$ is locally inverse. From [8, Theorem 7.6], it thus follows that in $S / \rho_{i}$ sandwich sets are singletons. From the above we infer $h_{i-1} \rho_{i}=h_{i} \rho_{i}$. Hence there exists a sequence

$$
e=h_{0} \rho_{1} h_{1} \rho_{2} \cdots \rho_{n-1} h_{n-1} \rho_{n} h_{n}=f,
$$

thus $(e, f) \in \operatorname{tr} \bigvee_{\rho \in \mathcal{F}} \rho$. We conclude that the equality $\bigvee_{\rho \in \mathcal{F}} \operatorname{tr} \rho=\operatorname{tr} \bigvee_{\rho \in \mathcal{F}} \rho$ holds.

Suppose next that $S$ is group bound. For $i \in\{0, \ldots, n\}$, let $m_{i}$ be a positive integer such that $x_{i}^{m_{i}}$ belongs to a maximal subgroup of $S$. Let $m=\max \left\{m_{0}, \ldots, m_{n}\right\}$. Then $x_{i}^{m}$ belongs to a maximal subgroup of $S$ for every $i=0, \ldots, n$, and $x_{i-1}^{m} \rho_{i} x_{i}^{m}$ for each $i=1, \ldots, n$. Let $e_{i}$ be the identity element of the maximal subgroup to which $x_{i}^{m}$ belongs. Then $x_{i-1}^{m} \rho_{i} x_{i}^{m}$ implies $e_{i-1} \rho_{i} e_{i}$. Further $e_{0}=x_{0}^{m}=e^{m}=e$ and $e_{n}=x_{n}^{m}=f^{m}=f$. Hence there exists a sequence

$$
e=e_{0} \rho_{1} e_{1} \rho_{2} e_{2} \cdots \rho_{n-1} e_{n-1} \rho_{n} e_{n}=f
$$

and we again conclude that $\bigvee_{\rho \in \mathcal{F}} \operatorname{tr} \rho=\operatorname{tr} \bigvee_{\rho \in \mathcal{F}} \rho$ holds.

We finally consider the case where $S$ orthodox. Let $x_{i}^{\prime} \in V\left(x_{i}\right), i=0, \ldots, n$. Then for $1<i \leq n$ we find $\left(x_{i-1}^{\prime} x_{i-1} x_{i}^{\prime} x_{i}\right) \rho_{i}\left(x_{i-1}^{\prime} x_{i-1}\right)$ since $\left(x_{i-1}^{\prime} x_{i-1}\right) \rho_{i}$ and $\left(x_{i}^{\prime} x_{i}\right) \rho_{i}$ are $\mathcal{L}$-related idempotents in $S / \rho_{i}$. Therefore

$$
\begin{aligned}
& x_{0}^{\prime} x_{0} \rho_{1} x_{0}^{\prime} x_{0} x_{1}^{\prime} x_{1} \rho_{2} x_{0}^{\prime} x_{0} x_{1}^{\prime} x_{1} x_{2}^{\prime} x_{2} \rho_{3} \\
& \quad \cdots \rho_{n} x_{0}^{\prime} x_{0} x_{1}^{\prime} x_{1} x_{2}^{\prime} x_{2} \cdots x_{n-2}^{\prime} x_{n-2} x_{n-1}^{\prime} x_{n-1} x_{n}^{\prime} x_{n} \\
& \quad \rho_{n-1} x_{0}^{\prime} x_{0} x_{1}^{\prime} x_{1} x_{2}^{\prime} x_{2} \cdots x_{n-2}^{\prime} x_{n-2} x_{n}^{\prime} x_{n} \\
& \quad \cdots \rho_{2} x_{0}^{\prime} x_{0} x_{1}^{\prime} x_{1} x_{n}^{\prime} x_{n} \rho_{1} x_{0}^{\prime} x_{0} x_{n}^{\prime} x_{n}
\end{aligned}
$$


so

$$
x_{0}^{\prime} x_{0}\left(\bigvee_{\rho \in \mathcal{F}} \operatorname{tr} \rho\right) x_{0}^{\prime} x_{0} x_{n}^{\prime} x_{n}
$$

Dually, from $\left(x_{i-1} x_{i-1}^{\prime} x_{i} x_{i}^{\prime}\right) \rho_{i}\left(x_{i} x_{i}^{\prime}\right)$ for all $1<i \leq n$ we find

$$
x_{n} x_{n}^{\prime}\left(\bigvee_{\rho \in \mathcal{F}} \operatorname{tr} \rho\right) x_{0} x_{0}^{\prime} x_{n} x_{n}^{\prime}
$$

If in particular $x_{0}^{\prime}=e$ and $x_{n}^{\prime}=f$ the above expressions yield

$$
e\left(\bigvee_{\rho \in \mathcal{F}} \operatorname{tr} \rho\right) \text { ef }\left(\bigvee_{\rho \in \mathcal{F}} \operatorname{tr} \rho\right) f
$$

thus $e\left(\bigvee_{\rho \in \mathcal{F}} \rho\right) f$. We conclude that $\operatorname{tr}\left(\bigvee_{\rho \in \mathcal{F}} \rho\right)=\bigvee_{\rho \in \mathcal{F}} \operatorname{tr} \rho$.

5. Properties of traces and kernels. In this section we investigate the $T_{l^{-}}$, $T_{r^{-}}, T$ - and $K$-classes of a congruence $\rho$. As we shall see, the classes of the first three congruences exhibit many nice properties. It appears that not much could be said about $K$-classes. We first consider the traces.

5.1. THEOREM $[11, \S 3]$. Let $\rho$ be a congruence on $S$. Then $\rho T$ consists of commuting congruences.

PROOF. This is immediate from Theorem 4.15.

5.2. CoROllaRY [11, THEOREM 3.4]. If $\rho$ is a congruence on $S$, then $\rho T$ is a complete modular lattice.

Proof. This is immediate from Theorem 5.1 and [1, Corollary 1 to IV.9].

Later in this section we shall show another way to derive the above result. We first introduce some new concepts.

5.3. DEFinition. A regular semigroup $S$ is left [right] fundamental if $\varepsilon$ is the only congruence on $S$ contained in $\mathcal{L}[R] ; S$ is fundamental if $\varepsilon$ is the only congruence on $S$ contained in $H$.

5.4. NOTATION. Let

$$
\mu_{l}=\varepsilon^{T_{l}}, \quad \mu_{r}=\varepsilon^{T_{r}}, \quad \mu=\varepsilon^{T}
$$

In order to avoid confusion we shall sometimes use the notation $\mu_{S}, \mu_{l S}, \mu_{r S}$.

5.5. LEMMA. The following hold in $S$.

(i) $\mu_{l}=\mathcal{L}^{0}, \mu_{r}=\mathcal{R}^{0}, \mu=\not^{0}$.

(ii) $\mu=\mu_{l} \cap \mu_{r}$.

(iii) $S$ is left [right fundamental if and only if $\varepsilon=\mathcal{L}^{0}\left[\varepsilon=R^{0}\right]$.

(iv) $S$ is fundamental if and only if $\varepsilon=\not^{0}$.

PROOF. That $\mu_{l}=\mathcal{L}^{0}$ and $\mu_{r}=R^{0}$ follows immediately from Theorem 4.12 and its dual. Theorem 4.14 gives

$$
\mu=\varepsilon^{T}=\varepsilon^{T_{l}} \cap \varepsilon^{T_{r}}=\mu_{l} \cap \mu_{r}=\mathcal{L}^{0} \cap R^{0}=(\mathcal{L} \cap R)^{0}=\mathscr{H}^{0} .
$$

The remaining statements are obvious from the definitions. 
We remark that from Lemma 2.8 it follows that $\mu=\not \gamma^{0}$ is the greatest congruence on $S$ with trace the equality on $E(S)$. In other words $\mu$ is the greatest idempotent separating congruence on $S$. Accordingly, $S$ is fundamental if and only if $\varepsilon$ is the only idempotent separating congruence on $S$.

5.6. LEMMA. For a congruence $\rho$ on $S$ we have

$$
\rho^{T_{r}} / \rho=\mu_{r S / \rho}, \quad \rho^{T} / \rho=\mu_{S / \rho}
$$

PROOF. If $a \rho^{T_{r}} b$, then $a R(\operatorname{tr} \rho) R(\operatorname{tr} \rho) R b$ by Lemma 4.3 and Theorem 4.12. Thus there exist $e, f, g, h \in E(S)$ such that $a R e(\operatorname{tr} \rho) f R g(\operatorname{tr} \rho) h R b$, whence $(a \rho) R(e \rho)=(f \rho) R(g \rho)=(h \rho) R(b \rho)$. We have proved that

$$
(a \rho)\left(\rho^{T_{r}} / \rho\right)(b \rho) \Rightarrow(a \rho) R(b \rho) .
$$

Consequently $\rho^{T_{r}} / \rho \subseteq \mu_{r S / \rho}$.

Conversely, if $(a \rho) \mu_{r S / \rho}(b \rho)$, choose $e \in E\left(R_{a}\right), f \in E\left(R_{b}\right)$ and $g \in S(e, f)$. Then $(e \rho) R(a \rho) R(b \rho) R(f \rho)$ in $S / \rho$ and thus

$$
h \rho \in S(e, f) \rho \subseteq S(e \rho, f \rho)=e \rho
$$

by Result 1.1 and

$$
(h f) \rho=(h \rho)(f \rho)=(e \rho)(f \rho)=f \rho .
$$

We thus find $a R e(\operatorname{tr} \rho) h R h f(\operatorname{tr} \rho) f R b$. Consequently, if $\theta$ is the congruence on $S$ given by

$$
a \theta b \Leftrightarrow(a \rho) \mu_{r S / \rho}(b \rho),
$$

then $\theta \subseteq R(\operatorname{tr} \rho) R(\operatorname{tr} \rho) R$. It follows that $\theta \subseteq(R(\operatorname{tr} \rho) R(\operatorname{tr} \rho) R)^{0}=\rho^{T_{r}}$, where the latter equality follows from Lemma 4.3 and Theorem 4.12. Therefore

$$
\mu_{r S / \rho}=\theta / \rho \subseteq \rho^{T_{r}} / \rho
$$

and the required equality prevails. Hence also

$$
\begin{aligned}
\mu_{S / \rho} & \left.=\mu_{l S / \rho} \cap \mu_{r S / \rho} \quad \text { (by Lemma } 5.5(i i)\right) \\
& =\left(\rho^{T_{l}} / \rho\right) \cap\left(\rho^{T_{r}} / \rho\right) \quad \text { (by the first part of the proof and its dual) } \\
& =\left(\rho^{T_{l}} \cap \rho^{T_{r}}\right) / \rho=\rho^{T} / \rho \quad \text { (by Theorem 4.14). }
\end{aligned}
$$

5.7. COROLLARY. Let $\rho$ be a congruence on $S$. Then $\rho=\rho^{T_{r}}$ if and only if $S / \rho$ is right fundamental; $\rho=\rho^{T}$ if and only if $S / \rho$ is fundamental.

PROOF. This follows easily from Lemmas 5.5 and 5.6.

5.8. Proposition. For any congruence $\rho$ on $S$, the mapping

$$
\varphi: \theta \rightarrow \theta / \rho_{T_{r}} \quad\left(\theta \in\left[\rho_{T_{r}}, \rho^{T_{r}}\right]\right)
$$

is an isomorphism of $\left[\rho_{T_{r}}, \rho^{T_{r}}\right]$ onto $\left[\varepsilon_{S / \rho_{T_{r}}}, \mu_{r S / \rho_{T_{r}}}\right]$.

PROOF. The mapping $\varphi$ is the restriction to $\left[\rho_{T_{r}}, \rho^{T_{r}}\right]$ of the isomorphism $\theta \rightarrow$ $\theta / \rho_{T_{r}}$ of $\left[\rho_{T_{r}}, \omega\right]$ onto Con $S / \rho_{T_{r}}$. Obviously $\left(\rho_{T_{r}}\right)^{T_{r}}=\rho^{T_{r}}$ so that by Lemma 5.6, $\rho^{T_{r}} / \rho_{T_{r}}=\mu_{r S} / \rho_{T_{r}}$. One now shows easily that $\varphi$ is a bijection of $\left[\rho_{T_{r}}, \rho^{T_{r}}\right]$ onto $\left[\varepsilon_{S / \rho_{T_{r}}}, \mu_{r S / \rho_{T_{r}}}\right]$ and thus also an isomorphism of complete lattices.

The proof of the next result follows along the same lines. 
5.9. PROPOSITION. For any congruence $\rho$ on $S$ the mapping

$$
\varphi: \theta \rightarrow \theta / \rho_{T} \quad\left(\theta \in\left[\rho_{T}, \rho^{T}\right]\right)
$$

is an isomorphism of $\left[\rho_{T}, \rho^{T}\right]$ onto $\left[\varepsilon_{S / \rho_{T}}, \mu_{S / \rho_{T}}\right]$.

5.10. Proposition. For each $e \in E(S)$, let $\mathfrak{N}_{e}$ be the lattice of normal subgroups of $H_{e}$. Then the mapping

$$
\varphi: \rho \rightarrow(e \rho, e \in E(S)) \quad(\rho \in[\varepsilon, \mu])
$$

is a complete monomorphism of $[\varepsilon, \mu]$ into $\prod_{e \in E(S)} \mathfrak{N}_{e}$.

PRoof. If $\rho \in[\varepsilon, \mu]$, then $\rho \subseteq \not$, so $e \rho \subseteq H_{e}$ for each $e \in E(S)$. Since $\left.\rho\right|_{H_{e}}$ is a congruence on the group $H_{e}, e \rho$ is a normal subgroup of $H_{e}$. Hence

$$
(e \rho, e \in E(S)) \in \prod_{e \in E(S)} \mathfrak{N}_{e}
$$

Let $\mathcal{F}$ be a family of congruences in $[\varepsilon, \mu]$. For $e \in E(S), e\left(\bigcap_{\rho \in \mathcal{F}} \rho\right)=\bigcap_{\rho \in \mathcal{F}} e \rho$, and it follows that $\varphi$ is a complete $\bigcap$-homomorphism. Let $x \in e\left(\bigvee_{\rho \in \mathcal{F}} \rho\right), e \in E(S)$. Then there exist $\rho_{1}, \ldots, \rho_{n} \in \mathcal{F}$ and $x_{0}, \ldots, x_{n} \in S$ such that $e=x_{0} \rho_{1} x_{1} \rho_{2} x_{2} \ldots$ $\rho_{n} x_{n}=x$. Since $\rho_{i} \subseteq \mathcal{H}$ for all $i$, we have that $x_{0}, \ldots, x_{n} \in H_{e}$ and $e=$ $x_{0}\left(\left.\rho_{1}\right|_{H_{e}}\right) x_{1}\left(\left.\rho_{2}\right|_{H_{e}}\right) x_{2} \cdots\left(\left.\rho_{n}\right|_{H_{e}}\right) x_{n}=x$ in the group $H_{e}$. Consequently $x \in$ $\bigvee_{\rho \in \mathcal{F}} e \rho$. Conversely if $x \in \bigvee_{\rho \in \mathcal{F}} e \rho$, then $x$ is $\left(\bigvee_{\rho \in \mathcal{F}} \rho\right)$-related to $e$ in the group $H_{e}$ and thus also in $S$. We conclude that for $e \in E(S), e\left(\bigvee_{\rho \in \mathcal{F}} \rho\right)=\bigvee_{\rho \in \mathcal{F}} e \rho$. Hence $\varphi$ is also a complete $\bigvee$-homomorphism.

If $\rho$ and $\theta$ are different congruences in $[\varepsilon, \mu]$, then from $\operatorname{tr} \rho=\left.\varepsilon\right|_{E(S)}=\operatorname{tr} \theta$ and Lemma 1.2 it follows that $\operatorname{ker} \rho \neq \operatorname{ker} \theta$. Consequently there exists $e \in E(S)$ such that $e \rho \neq e \theta$. Therefore $\varphi$ is injective.

5.11. COROLLARY. The lattice $[\varepsilon, \mu]$ of idempotent separating congruences is a complete modular lattice.

PROOF. Since the lattice of normal subgroups of a group is modular, and since by Proposition $5.10[\varepsilon, \mu]$ is embeddable into the lattice of normal subgroups of a group, we conclude that $[\varepsilon, \mu]$ is modular.

5.12. COROllaRY [11, THEOREM 3.4]. If $\rho$ is a congruence on $S$, then $\left[\rho_{T}, \rho^{T}\right]$ is a complete modular lattice.

PROOF. This is immediate from Theorem 5.9 and Corollary 5.11.

We now turn to the properties of the congruence $K$.

5.13. EXAMPLE $[9, \S 3]$. Let $S$ be the direct product of a nontrivial group and a two-element semilattice. Then the relation $K$ on $\operatorname{Con} S$ is not a congruence.

We nevertheless have the following.

5.14. Proposition. Let $\rho$ be a congruence on $S$. Then $\rho K=\left[\rho_{K}, \rho^{K}\right]$ is a complete sublattice of $\operatorname{Con} S$.

PROOF. That $\rho K$ is an interval follows from Lemmas 2.4 and 2.9. The remaining part in the statement is clear from the fact that an interval of a complete lattice is a complete sublattice.

5.15. Definition. A congruence $\rho$ on $S$ is idempotent pure if $\operatorname{ker} \rho=E(S)$. Furthermore, $S$ is $E$-disjunctive if $\varepsilon$ is the only idempotent pure congruence on $S$. 

$\left[\varepsilon, \varepsilon^{K}\right]$.

5.16. LEMMA. A congruence $\rho$ on $S$ is idempotent pure if and only if $\rho \in$

ProOF. If $\rho \in\left[\varepsilon, \varepsilon^{K}\right]$, then $\operatorname{ker} \rho \subseteq \operatorname{ker} \varepsilon^{K}=E(S)$ implies $\operatorname{ker} \rho=E(S)$. Conversely, if $\rho$ is idempotent pure, then $\rho \subseteq \varepsilon^{K}$ since $\varepsilon^{K}$ is the greatest congruence with $\operatorname{kernel} \operatorname{ker} \varepsilon=E(S)=\operatorname{ker} \rho$.

5.17. LEMMA. For a congruence $\rho$ on $S$ we have

$$
\rho^{K} / \rho=\left(\varepsilon_{S / \rho}\right)^{K} \text {. }
$$

PROOF. If $a \rho \in \operatorname{ker}\left(\rho^{K} / \rho\right)$, then $(a \rho)\left(\rho^{K} / \rho\right)(a \rho)^{2}=a^{2} \rho$ and thus $a \rho^{K} a^{2}$. From this we infer that $a \in \operatorname{ker} \rho^{K}=\operatorname{ker} \rho$ and consequently that $a \rho \in E(S / \rho)$. We conclude that $\operatorname{ker}\left(\rho^{K} / \rho\right)=E(S / \rho)=\operatorname{ker}\left(\varepsilon_{S / \rho}\right)^{K}$ and thus $\rho^{K} / \rho \subseteq\left(\varepsilon_{S / \rho}\right)^{K}$.

Let $a \rho, b \rho \in S / \rho$ be such that $(a \rho)\left(\varepsilon_{S / \rho}\right)^{K}(b \rho)$. In view of Lemma 2.4 we have for all $x, y \in S^{1}$,

$$
(x \rho)(a \rho)(y \rho) \in E(S / \rho) \Leftrightarrow(x \rho)(b \rho)(y \rho) \in E(S / \rho),
$$

or in other words, because of Result 1.2 , we obtain that

$$
x a y \in \operatorname{ker} \rho \Leftrightarrow x b y \in \operatorname{ker} \rho .
$$

Therefore we have

$$
(a \rho)\left(\varepsilon_{S / \rho}\right)^{K}(b \rho) \Rightarrow a \pi_{\text {ker } \rho} b \Leftrightarrow a \rho^{K} b .
$$

Consequently, $\left(\varepsilon_{S / \rho}\right)^{K} \subseteq \rho^{K} / \rho$.

5.18. COROLLARY. Let $\rho$ be a congruence on $S$. Then $\rho=\rho^{K}$ if and only if $S / \rho$ is E-disjunctive.

PROOF. This follows easily from Lemmas 5.16 and 5.17.

The proof of the next result follows along the same lines as the proof of Proposition 5.8 .

5.19. Proposition. For any congruence $\rho$ on $S$, the mapping

$$
\varphi: \theta \rightarrow \theta / \rho_{K} \quad\left(\theta \in\left[\rho_{K}, \rho^{K}\right]\right)
$$

is an isomorphism of $\left[\rho_{K}, \rho^{K}\right]$ onto $\left[\varepsilon_{S / \rho},\left(\varepsilon_{S / \rho}\right)^{K}\right]$.

6. Congruence triples. In the light of the results obtained in $\S 4$ we are now able to present a refinement of the concept of congruence pairs of $\S 2$. We arrive here at congruence triples for a regular semigroup which are analogous to admissible triples used for describing congruences on Rees matrix semigroups (see [5, III.4]).

6.1. Notation. Let $S$ be a regular semigroup, $\gamma \in \operatorname{Eq}(S / \mathcal{L})$ and $\delta \in \operatorname{Eq}(S / R)$. For any $a, b \in S$, we put

$$
a \bar{\gamma} b \Leftrightarrow L_{a} \gamma L_{b}, \quad a \bar{\delta} b \Leftrightarrow R_{a} \delta R_{b} .
$$

It is evident that $\bar{\gamma}, \bar{\delta} \in \operatorname{Eq} S$, where $\mathcal{L} \subseteq \bar{\gamma}$ and $R \subseteq \bar{\delta}$.

We introduce next the following concepts.

6.2. Definition. An equivalence $\gamma \in \operatorname{Eq}(S / \mathcal{L})$ is normal if

$$
\gamma=\left(\bar{\gamma}^{0} \vee \mathcal{L}\right) / \mathcal{L}
$$

and an equivalence $\delta \in \operatorname{Eq}(S / R)$ is normal if

$$
\delta=\left(\bar{\delta}^{0} \vee R\right) / R \text {. }
$$

If $\rho$ is a congruence on $S$, then $(\rho \vee \mathcal{L}) / \mathcal{L}[(\rho \vee R) / R]$ is the $\mathcal{L}$-part [R-part] of $\rho$. 
6.3. LEMMA. An equivalence $\delta \in \mathrm{Eq}(S / R)$ is normal if and only if $\delta$ is the $R$-part of a congruence $\rho$ on $S$.

PROOF. If $\delta$ is normal, then $\delta$ is the $R$-part of the congruence $\bar{\delta}^{0}$. Suppose conversely that $\delta$ is the $R$-part of a congruence $\rho$, that is $\delta=(\rho \vee R) / R$, or in other words, $\bar{\delta}=\rho \vee R$. Clearly $\rho \subseteq \bar{\delta}^{0}$ so that $\rho \vee R \subseteq \bar{\delta}^{0} \vee R$. On the other hand $\bar{\delta}^{0} \subseteq \rho \vee R$ and thus $\bar{\delta}^{0} \vee R \subseteq \rho \vee R$. We therefore have $\delta=(\rho \vee R) / R=\left(\bar{\delta}^{0} \vee R\right) / R$ and $\delta$ is normal.

Our final basic concept is the following.

6.4. DEFINITION. A triple $(\gamma, K, \delta)$ consisting of normal equivalences $\gamma \in$ $\operatorname{Eq}(S / \mathcal{L})$ and $\delta \in \operatorname{Eq}(S / R)$ and a normal subset $K \subseteq S$, is a congruence triple if

(i) $\bar{\gamma}=(\bar{\gamma} \cap \bar{\delta})^{0} \vee \mathcal{L}, \bar{\delta}=(\bar{\gamma} \cap \bar{\delta})^{0} \vee R$,

(ii) $K \subseteq \operatorname{ker} \bar{\gamma}^{0}, \bar{\gamma} \subseteq \theta_{K}^{0} \vee \mathcal{L}$,

(iii) $K \subseteq \operatorname{ker} \bar{\delta}^{0}, \bar{\delta} \subseteq \theta_{K}^{0} \vee R$.

If this is the case, we define

$$
\rho_{(\gamma, K, \delta)}=\left(\bar{\gamma} \cap \theta_{K} \cap \bar{\delta}\right)^{0} .
$$

For the principal result of this section, we prove first an auxiliary result which is also of independent interest (cf. Theorem 4.7).

\subsection{LEMMA. The mapping}

$$
\rho \rightarrow \rho \vee R \quad(\rho \in \operatorname{Con} S)
$$

is a complete homomorphism of Con $S$ into $\operatorname{Eq} S$ which induces $T_{r}$.

ProOF. Let $₹$ be a family of congruences on $S$. Let $a, b \in S$ be such that $a\left(\bigcap_{\rho \in \mathcal{F}}(\rho \vee R)\right) b$. It follows that $a(\rho \vee R) b$ for every $\rho \in \mathcal{F}$. If $e \in E\left(R_{a}\right)$ and $f \in E\left(R_{b}\right)$, then the foregoing implies that $e(\rho \vee R) f$ for every $\rho \in \mathcal{F}$. Hence $e\left(\bigcap_{\rho \in \mathcal{F}}(\rho \vee R)\right) f$. Applying Theorem 4.7 we have $e\left(\left(\bigcap_{\rho \cap \mathcal{F}} \rho\right) \vee R\right) f$ and thus also $\left.a\left(\left(\bigcap_{\rho \in \mathcal{F}}\right) \rho\right) \vee R\right) b$. We have proved that

$$
\bigcap_{\rho \in \mathcal{F}}(\rho \vee R) \subseteq\left(\bigcap_{\rho \in \mathcal{F}} \rho\right) \vee R .
$$

The reverse inclusion is obvious and so the equality prevails. It follows that the mapping $\rho \rightarrow \rho \vee R(\rho \in \operatorname{Con} S)$ is a complete lattice homomorphism of Con $S$ into $\operatorname{Eq} S$.

If $\rho, \theta \in \operatorname{Con} S$ are such that $\rho T_{r} \theta$, then $\operatorname{rtr} \rho=\operatorname{rtr} \theta$, so that, by Lemma 4.3(i),

$$
\rho \vee R=R(\operatorname{rtr} \rho) R(\operatorname{rtr} \rho) R=R(\operatorname{rtr} \theta) R(\operatorname{trt} \theta) R=\theta \vee R .
$$

Conversely if $\rho \vee R=\theta \vee R$, then

$$
\operatorname{rtr} \rho=\operatorname{tr}(\rho \vee R)^{0}=\operatorname{tr}(\theta \vee R)^{0}=\operatorname{rtr} \theta .
$$

The next result describes congruences on a regular semigroup in terms of congruence triples. It represents an analogue of Theorem 2.13 . 
6.6. THEOREM. If $(\gamma, K, \delta)$ is a congruence triple for $S$, then $\rho_{(\gamma, K, \delta)}$ is the unique congruence $\rho$ on $S$ such that $\gamma$ is the $\mathcal{L}$-part of $\rho, K=\operatorname{ker} \rho$ and $\delta$ is the R-part of $\rho$. Conversely, if $\rho$ is a congruence on $S$, then $(\gamma, K, \delta)=$ $((\rho \vee \mathcal{L}) / \mathcal{L}, \operatorname{ker} \rho,(\rho \vee \mathcal{R}) / \mathcal{R})$ is a congruence triple for $S$ and $\rho=\rho_{(\gamma, K, \delta)}$.

Proof. Let $(\gamma, K, \delta)$ be a congruence triple. Then

$$
\begin{aligned}
\operatorname{ker} \rho_{(\gamma, K, \delta)} & =\operatorname{ker}\left(\bar{\gamma} \cap \theta_{K} \cap \bar{\delta}\right)^{0} \\
& =\operatorname{ker}\left(\bar{\gamma}^{0} \cap \theta_{K}^{0} \cap \bar{\delta}^{0}\right) \quad(\text { by Result 1.4) } \\
& =\operatorname{ker} \bar{\gamma}^{0} \cap \operatorname{ker} \theta_{K}^{0} \cap \operatorname{ker} \bar{\delta}^{0} \quad(\text { by Lemma 2.5) } \\
& =\operatorname{ker} \bar{\gamma}^{0} \cap K \cap \operatorname{ker} \bar{\delta}^{0} \quad(\text { since } K \text { is a normal subset of } S) \\
& =K \quad\left(\text { since } K \subseteq \operatorname{ker} \bar{\gamma}^{0} \text { and } K \subseteq \operatorname{ker} \bar{\delta}^{0}\right) .
\end{aligned}
$$

Further,

$$
\begin{aligned}
\rho_{(\gamma, K, \delta)} \vee R & =\left(\bar{\gamma} \cap \theta_{K} \cap \bar{\delta}\right)^{0} \vee R \\
& =\left((\bar{\gamma} \cap \bar{\delta})^{0} \cap \theta_{K}^{0}\right) \vee R \quad \text { (by Result 1.4) } \\
& =\left((\bar{\gamma} \cap \bar{\delta})^{0} \vee R\right) \cap\left(\theta_{K}^{0} \vee R\right) \quad(\text { by Lemma 6.5) } \\
& =\bar{\delta} \cap\left(\theta_{K}^{0} \vee R\right) \quad\left(\text { since } \bar{\delta}=(\bar{\gamma} \cap \bar{\delta})^{0} \vee R\right) \\
& =\bar{\delta} \quad\left(\text { since } \bar{\delta} \subseteq \theta_{K}^{0} \vee R\right),
\end{aligned}
$$

and thus

$$
\left(\rho_{(\gamma, K, \delta)} \vee R\right) / R=\delta
$$

is the $R$-part of $\rho_{(\gamma, K, \delta)}$. Dually, $\gamma$ is the $\mathcal{L}$-part of $\rho_{(\gamma, K, \delta)}$.

Let $(\gamma, K, \delta)$ be a congruence triple and $\theta \in \operatorname{Con} S$ be such that $\gamma$ is the $\mathcal{L}$-part of $\theta, K=\operatorname{ker} \theta$ and $\delta$ is the $R$-part of $\theta$. Then $\operatorname{ker} \theta=K=\operatorname{ker} \rho_{(\gamma, K, \delta)}$. Further,

$$
\begin{aligned}
\operatorname{tr} \theta & =\operatorname{lt} \theta \cap \operatorname{rtr} \theta \quad(\text { by Lemma 4.3(iv)) } \\
& =\operatorname{tr}(\delta \vee \mathcal{L})^{0} \cap \operatorname{tr}(\theta \vee R)^{0}=\operatorname{tr} \bar{\gamma}^{0} \cap \operatorname{tr} \bar{\delta}^{0},
\end{aligned}
$$

and similarly

$$
\operatorname{tr} \rho_{(\gamma, K, \delta)}=\operatorname{tr} \bar{\gamma}^{0} \cap \operatorname{tr} \bar{\delta}^{0} .
$$

From Lemma 2.10 we may now conclude that $\theta=\rho_{(\gamma, K, \delta)}$.

Let $\rho \in \operatorname{Con} S$ and let $\gamma=(\rho \vee \mathcal{L}) / \mathcal{L}, K=\operatorname{ker} \rho$ and $\delta=(\rho \vee \mathcal{R}) / \mathcal{R}$. By Lemma 6.3 and its dual $\gamma$ and $\delta$ are normal equivalences on $\mathrm{Eq}(S / \mathcal{L})$ and $\mathrm{Eq}(S / R)$, respectively. By Lemma $2.4, K$ is a normal subset of $S$. We note that $\bar{\gamma}=\rho \vee \mathcal{L}$ and $\bar{\delta}=\rho \vee R$.

Since $R \subseteq \bar{\delta}$, it follows that $(\bar{\gamma} \cap \bar{\delta})^{0} \vee R \subseteq \bar{\delta}$. Further, since $\rho \subseteq(\bar{\gamma} \cap \bar{\delta})^{0}$ we have $\operatorname{tr} \rho \subseteq(\bar{\gamma} \cap \bar{\delta})^{0}$. This together with Lemma 2.6(iii) gives

$$
\bar{\delta}=\rho \vee R=R(\operatorname{tr} \rho) R(\operatorname{tr} \rho) R \subseteq(\bar{\gamma} \cap \bar{\delta})^{0} \vee R .
$$

Therefore $\bar{\delta}=(\bar{\gamma} \cap \bar{\delta})^{0} \vee R$ and dually, $\bar{\gamma}=(\bar{\gamma} \cap \bar{\delta})^{0} \vee \mathcal{L}$.

From $\rho \subseteq(\rho \vee R)^{0}=\bar{\delta}^{0}$ it immediately follows that $K=\operatorname{ker} \rho \subseteq \operatorname{ker} \bar{\delta}^{0}$. Further, $\operatorname{ker} \rho=K$ gives $\rho \subseteq \theta_{K}^{0}$ whence $\bar{\delta}=\rho \vee R \subseteq \theta_{K}^{0} \vee R$. A dual reasoning yields $K \subseteq \bar{\gamma}^{0}$ and $\bar{\gamma} \subseteq \theta_{K}^{0} \vee \mathcal{L}$.

We have proved that $(\gamma, K, \delta)$ is a congruence triple. From the first part of the proof we may now conclude that $\rho=\rho_{(\gamma, K, \delta)}$. 
6.7. Proposition. Let $\mathrm{Ct} S$ be the poset of all congruence triples for $S$ under the partial order given by

$$
(\gamma, K, \delta) \subseteq\left(\gamma^{\prime}, K^{\prime}, \delta^{\prime}\right) \Leftrightarrow \gamma \subseteq \gamma^{\prime}, K \subseteq K^{\prime}, \delta \subseteq \delta^{\prime} .
$$

Then the mappings

$$
\rho \rightarrow((\rho \vee \mathcal{L}) / \mathcal{L}, \operatorname{ker}, \rho,(\rho \vee \mathcal{R}) / \mathcal{R}), \quad(\gamma, K, \delta) \rightarrow \rho_{(\gamma, K, \delta)}
$$

are mutually inverse isomorphisms of $\mathrm{Con} S$ and $\mathrm{Ct} S$.

PROOF. This follows easily from Theorem 6.6.

As an immediate consequence of Lemma 6.5 and Theorem 6.6 we have

6.8. COROLLARY. The mapping

$$
\rho_{(\gamma, K, \delta)} \rightarrow \delta \quad((\gamma, K, \delta) \in \mathrm{Ct} S)
$$

is a complete homomorphism of $\operatorname{Con} S$ into $\mathrm{Eq}(S / R)$ inducing $T_{r}$. The normal equivalences on $S / R$ form a complete sublattice of $\mathrm{Eq}(S / R)$.

6.9. Proposition. Let $\left(\gamma_{i}, K_{i}, \delta_{i}\right), i=1,2$, be congruence triples for $S$. Let $\rho_{i}=\rho_{\left(\gamma_{i}, K_{i}, \delta_{i}\right)}, i=1,2$. Then

$$
\begin{aligned}
& \rho_{1} T_{l} \rho_{2} \Leftrightarrow \gamma_{1}=\gamma_{2}, \\
& \rho_{1} K \rho_{2} \Leftrightarrow K_{1}=K_{2}, \\
& \rho_{1} T_{r} \rho_{2} \Leftrightarrow \delta_{1}=\delta_{2} .
\end{aligned}
$$

PROOF. We have

$$
\begin{aligned}
\rho_{1} T_{r} \rho_{2} & \Leftrightarrow \rho_{1} \vee R=\rho_{2} \vee R \quad \text { (by Theorem 6.5) } \\
& \Leftrightarrow \bar{\delta}_{1}=\bar{\delta}_{2} \quad(\text { by Theorem 6.6) } \\
& \Leftrightarrow \delta_{1}=\delta_{2} .
\end{aligned}
$$

This together with its dual gives the first and the third part in the statement of the proposition in view of Corollary 4.8. The second part follows immediately from Theorem 6.6.

A simple expression for $\rho_{(\gamma, K, \delta)}$ is provided by the following.

6.10. Proposition. Let $(\gamma, K, \delta)$ be a congruence triple for $S$. Then for any $a, b \in S$,

$$
a \rho_{(\gamma, K, \delta)} b \Leftrightarrow L_{a} \gamma L_{b}, \quad R_{a} \delta R_{b} \text { and } a b^{\prime} \in K \text { for some }[\text { all }] b^{\prime} \in V(b) .
$$

ProOF. Let $\tau=\operatorname{tr} \rho_{(\gamma, K, \delta)}$. Then

$$
\begin{aligned}
\bar{\delta} & =\rho_{(\gamma, K, \delta)} \vee R \quad \text { (by Theorem 6.6) } \\
& =R \tau R \tau R \quad \text { (by Lemma 2.6(iii)). }
\end{aligned}
$$

Consequently, for $a, b \in S$ we have

$$
R_{a} \delta R_{b} \Leftrightarrow a(R \tau R \tau R) b
$$

and dually

$$
L_{a} \gamma L_{b} \Leftrightarrow a(\mathcal{L} \tau \mathcal{L} \tau \mathcal{L}) b .
$$

The result now follows from Lemma 2.10.

The following two results relate our representation of congruences by congruence triples with our findings of $\S 2$. 
6.11. Proposition. Let $\gamma \in \operatorname{Eq}(S / \mathcal{L})$ and $\delta \in \operatorname{Eq}(S / R)$ be normal equivalences such that 6.4(i) is satisfied. Then $\tau=\left.(\bar{\gamma} \cap \bar{\delta})\right|_{E(S)}=\operatorname{tr}(\bar{\gamma} \cap \bar{\delta})^{0}$ is a normal equivalence on $E(S)$ and

$$
\gamma=(\mathcal{L} \tau \mathcal{L} \tau \mathcal{L}) / \mathcal{L}, \quad \delta=(R \tau R \tau R) / R .
$$

Conversely, if $\tau$ is a normal equivalence on $E(S)$, then $\gamma=(\mathcal{L} \tau \mathcal{L} \tau \mathcal{L}) / \mathcal{L} \in$ $\operatorname{Eq}(S / \mathcal{L})$ and $\delta=(R \tau R \tau R) / R \in \operatorname{Eq}(S / R)$ are normal equivalences such that $6.4(\mathrm{i})$ is satisfied and $\tau=\left.(\bar{\gamma} \cap \bar{\delta})\right|_{E(S)}$.

PROOF. We first suppose that $\gamma$ and $\delta$ are normal equivalences satisfying 6.4(i). We put $\rho=(\bar{\gamma} \cap \bar{\delta})^{0}$ and $\tau=\operatorname{tr} \rho$. Then

$$
\begin{aligned}
\tau & =\tau \mathcal{L} \tau \cap \tau R_{\tau} \quad(\text { by Lemma 2.6(v)) } \\
& =\left.\left.(\rho \vee \mathcal{L})\right|_{E(S)} \cap(\rho \vee R)\right|_{E(S)} \quad \text { (by Lemma 2.6(iv)) } \\
& =\left.\left.\bar{\gamma}\right|_{E(S)} \cap \bar{\delta}\right|_{E(S)} \quad \text { (since 6.4(i) is satisfied) } \\
& =\left.(\bar{\gamma} \cap \bar{\delta})\right|_{E(S)} .
\end{aligned}
$$

We conclude that $\tau=\operatorname{tr}(\bar{\gamma} \cap \bar{\delta})^{0}=\left.(\bar{\gamma} \cap \bar{\delta})\right|_{E(S)}$ is a normal equivalence on $E(S)$ by Lemma 2.3.

If, conversely, $\tau$ is a normal equivalence on $E(S)$, then there exists $\rho \in \operatorname{Con} S$ such that $\tau=\operatorname{tr} \rho$. By Theorem $6.6 \gamma=(\rho \vee \mathcal{L}) / \mathcal{L}$ and $\delta=(\rho \vee R) / \tau$ are normal equivalences on $S / \mathcal{L}$ and $S / R$, respectively, such that condition 6.4(i) is satisfied. By Lemma 2.6(iii) we have $\gamma=(\mathcal{L} \tau \mathcal{L} \tau \mathcal{L}) / \mathcal{L}$ and $\delta=(R \tau R \tau R) / R$. Moreover,

$$
\begin{aligned}
\left.(\bar{\gamma} \cap \bar{\delta})\right|_{E(S)} & =\left.((\rho \vee \mathcal{L}) \cap(\rho \vee \mathcal{R}))\right|_{E(S)} \\
& =\left(\left.\left.(\rho \vee \mathcal{L})\right|_{E(S)} \cap(\rho \vee R)\right|_{E(S)}\right) \\
& =\tau \mathcal{L} \tau \cap \tau R \tau \quad \text { (by Lemma 2.6(iv) }) \\
& =\tau \quad(\text { by Lemma 2.6(v) }) .
\end{aligned}
$$

6.12. Proposition. Let $(\gamma, K, \delta)$ be a congruence triple. If $\tau=\left.(\bar{\gamma} \cap \bar{\delta})\right|_{E(S)}$, then $(K, \tau)$ is a congruence pair and $\rho_{(\gamma, K, \delta)}=\rho_{(K, \tau)}$.

Let $(K, \tau)$ be a congruence pair. If $\gamma=(\mathcal{L} \tau \mathcal{L} \tau \mathcal{L}) / \mathcal{L}$ and $\delta=(R \tau R \tau R) / R$, then $(\gamma, K, \delta)$ is a congruence triple and $\rho_{(K, \tau)}=\rho_{(\gamma, K, \delta)}$.

PROOF. Let $(\gamma, K, \delta)$ be a congruence triple. Then we can show as in the proof of Theorem 6.6 that

$$
\operatorname{tr} \rho_{(\gamma, K, \delta)}=\operatorname{tr} \bar{\gamma}^{0} \cap \operatorname{tr} \bar{\delta}^{0} .
$$

By Proposition 6.11 it thus follows that

$$
\tau=\left.(\bar{\gamma} \cap \bar{\delta})\right|_{E(S)}=\operatorname{tr}(\bar{\gamma} \cap \bar{\delta})^{0}=\operatorname{tr} \rho_{(\gamma, K, \delta)} .
$$

On the other hand $K=\operatorname{ker} \rho_{(\gamma, K, \delta)}$ by Theorem 6.6. By Theorem 2.13 we may conclude that $(K, \tau)$ is a congruence pair and that $\rho_{(\gamma, K, \delta)}=\rho_{(K, \tau)}$.

Conversely, let $(K, \tau)$ be a congruence pair. Then

$$
\begin{aligned}
\gamma & =(\mathcal{L} \tau \mathcal{L} \tau \mathcal{L}) / \mathcal{L} \\
& =\left(\mathcal{L}\left(\operatorname{tr} \rho_{(K, \tau)}\right) \mathcal{L}\left(\operatorname{tr} \rho_{(K, \tau)}\right) \mathcal{L}\right) / \mathcal{L} \quad(\text { by Theorem } 2.13) \\
& =\left(\rho_{(K, \tau)} \vee \mathcal{L}\right) / \mathcal{L} \quad(\text { by Lemma } 2.6(\text { iii }))
\end{aligned}
$$


and similarly

$$
\delta=\left(\rho_{(K, \tau)} \vee R\right) / R
$$

whereas

$$
K=\operatorname{ker} \rho_{(K, \tau)}
$$

by Theorem 2.13. By Theorem 6.6 we have that $(\gamma, K, \delta)$ is a congruence triple and that $\rho_{(\gamma, K, \delta)}=\rho_{(K, \tau)}$.

\section{REFERENCES}

1. G. Birkhoff, Lattice theory, Amer. Math. Soc. Colloq. Publ., vol. 25, Amer. Math. Soc., Providence, R. I., 1940.

2. A. H. Clifford, and G. B. Preston, The algebraic theory of semigroups, Math. Surveys, no. 7, Amer. Math. Soc., Providence, R. I., Vol. I, 1961, Vol. II, 1967.

3. R. Feigenbaum, Regular semigroup congruences, Semigroup Forum 17 (1979), 373-377.

4. T. E. Hall, On the lattice of congruences on a regular semigroup, Bull. Austral. Math. Soc. 1 (1969), 231-235.

5. J. M. Howie, An introduction to semigroup theory, Academic Press, New York, 1976.

6. G. Lallement, Demi-groupes réguliers, Ann. Mat. Pura Appl. 77 (1967), 47-129.

7. W. D. Munn, Decompositions of the congruence lattice of a semigroup, Proc. Edinburgh Math. Soc. 23 (1980), 193-198.

8. K. S. S. Nambooripad, Structure of regular semigroups. I, Mem. Amer. Math. Soc. No. 224 (1979).

9. F. Pastijn and P. Trotter, Lattices of completely regular semigroup varieties, Pacific J. Math. 119 (1985), 191-214.

10. M. Petrich, Inverse semigroups, Wiley, New York, 1984.

11. N. R. Reilly and H. E. Scheiblich, Congruences on regular semigroups, Pacific J. Math. 23 (1967), 349-360.

12. H. E. Scheiblich, Certain congruence and quotient lattices related to completely 0-simple and primitive regular semigroups, Glasgow Math. J. 10 (1969), 21-24.

13. __ Kernels of inverse semigroups homomorphisms, J. Austral. Math. Soc. 18 (1974), 289-292.

14. P. G. Trotter, Normal partitions of idempotents of regular semigroups, J. Austral. Math. Soc. A26 (1978), 110-114.

Dienst Hogere Meetkunde, Rijksuniversiteit te Gent, B-9000 Gent, Belgium

Current address (Francis Pastijn): Department of Mathematics, Statistics and Computer Science, Marquette University, Milwaukee, Wisconsin 53233 